\title{
THE INTERFACE CONTROL DOMAIN DECOMPOSITION (ICDD) METHOD FOR STOKES-DARCY COUPLING
}

\author{
MARCO DISCACCIATI ${ }^{\dagger}$, PAOLA GERVASIO ${ }^{\ddagger}$, ALESSANDRO GIACOMINI $^{\ddagger}$, AND ALFIO \\ QUARTERONI
}

\begin{abstract}
The ICDD method [15, 16] is here proposed to solve the coupling between Stokes and Darcy equations. According to this approach, the problem is formulated as an optimal control problem whose control variables are the traces of the velocity and the pressure on the internal boundaries of the subdomains that provide an overlapping decomposition of the original computational domain. A theoretical analysis is carried out and the well-posedness of the problem is proved under certain assumptions on both the geometry and the model parameters. An efficient solution algorithm is proposed, and several numerical tests are implemented. Our results show the accuracy of the ICDD method, its computational efficiency and robustness with respect to the different parameters involved (grid-size, polynomial degrees, permeability of the porous domain, thickness of the overlapping region). The ICDD approach turns out to be more versatile and easier to implement than the celebrated model based on the Beavers, Joseph and Saffman coupling conditions.
\end{abstract}

Key words. Domain decomposition, Optimal control, Stokes-Darcy coupling, Heterogeneous problems, ICDD.

AMS subject classifications. 49K20, 65N30, 65N35, 65N55, 76D07, 76S05

1. Introduction. Flow processes in a free-fluid region adjacent to a porous medium occur in many relevant applications: in the filtration of blood through arterial vessel walls and/or body tissues, in industrial processes involving, e.g., air or oil filters and fuel cells, in cross-flow filtration procedures, in the percolation of waters of hydrological basins through rocks and sand, just to mention a few.

At the microscopic scale the complete process can in principle be modelled by the Navier-Stokes equations in the whole domain (both in the free-fluid and in the porous medium regions). This would allow the computation of the exact velocity and stress fields without resorting to any averaging procedure. However, it would require a detailed description of the porous medium and its computational cost could be prohibitive.

Alternatively, under the (realistic) assumption that the Reynolds number in the porous domain is small, the Navier-Stokes equations could be therein upscaled to a macroscopic level and replaced by the Darcy law [12], the simplest linear relation between the fluid velocity and the pressure. Following this approach, the two different flow regimes (the free fluid and the porous-medium flow) must be suitably coupled to correctly describe the physical process of filtration, through transition region. An extensive overview of coupling strategies to be used in this transition layer is presented in $[28,40,41]$.

Physical considerations induce to speculate that the thickness of the transition region is $\mathcal{O}(\varepsilon)$, being $\varepsilon$ the characteristic length of the pores inside the porous medium. However, in several models such a region is replaced by a virtual (dimensionless) sharp interface.

\footnotetext{
${ }^{\dagger}$ Department of Mathematical Sciences, Loughborough University, LE11 3TU Loughborough, UK (m.discacciati@lboro.ac.uk)

¥DICATAM, Università degli Studi di Brescia, via Branze 38, I-25123 Brescia, Italy (paola.gervasio@unibs.it, alessandro.giacomini@unibs.it)

$\S$ MATHICSE, Chair of Modelling and Scientific Computing, Ecole Polytechnique Fédérale de Lausanne, Station 8, CH-1015 Lausanne, Switzerland (alfio.quarteroni@epfl.ch)
} 
In the latter case, the most popular method used to couple the two regimes consists in imposing a set of three interface conditions based on the mass conservation, the balance of normal forces, and the well-known Beavers-Joseph-Saffman (BJS) condition. The BJS condition was derived from experimental observations by Beavers and Joseph [1], then simplified by Saffmann [46] and later justified mathematically by Jäger and Mikelić $[29,30]$ by using homogenization techniques. Other approaches to derive the same condition are based on volume averaging, upscaling, or matched asymptotic expansion techniques (see, e.g., [40, 41, 31, 8]). However, since the BJS condition depends on a coefficient related to the structure of the porous material close to the interface region and to the position of the interface itself, it is not straightforward to be characterized (see, e.g. [30, 47, 7]).

Another approach, alternative to using interface conditions, consists in solving the Brinkman equation [3] in the whole domain (that is both in the fluid and in the porous medium). The transition between the two fluid regimes is achieved automatically by varying in a discontinuous way the permeability and the porosity across a sharp interface and by introducing the so-called effective viscosity in the porous medium. No interface conditions are needed in this approach. See [9] and references therein. This technique is very simple to implement and it is widely used in commercial software, however it is very sensitive to the parameters used in the model and some of the quantities involved (e.g., the effective viscosity) are difficult to characterize in practical applications [26, 27, 33].

Minor attention has been given in the literature to the case of a transition region with positive and small thickness. In [40, 41], Ochoa-Tapia and Whitaker solve the Stokes equations in the free-fluid domain, the Stokes problem with an additional term featuring a variable porosity inside the transition region, and the Darcy model with Brinkman correction in the porous domain. Their approach produces a jump in the stress but not in the velocity at the two interfaces of the transition region.

In this paper we propose an alternative approach still based on the use of a thin transition region. However, the novelty is that we do not solve any particular new equation inside such a region (neither we enforce any matching conditions in it). In fact, our transition region merely represents the overlap of two regions, one in which we solve the free fluid (Stokes) equations, the other where we solve the porous medium (Darcy) equation. The coupling between the two solutions is not modelled, rather it is achieved by imposing in a least-squares sense the continuity of velocity and pressure only across the two subdomain interfaces that delimitate the thin transition layer.

More specifically, we use the so-called Interface Control Domain Decomposition (ICDD) method, formerly introduced in [15] as a solution strategy for boundary value problems governed by elliptic partial differential equations and then extended to the Stokes equations in [16]. The ICDD method shares some similarities with the classic overlapping Schwarz method [44] and with the Least Square Conjugate Gradient [23] and the Virtual Control [37, 20,14] methods, and it is characterized by a decomposition of the original domain into overlapping regions.

Generally speaking, the ICDD method introduces new auxiliary control variables on the subdomain internal boundaries (named interfaces) that play the role of the unknown traces (or fluxes) of the state solutions of the subproblems. Such controls are determined by minimizing a suitable cost functional that measures the jump of the quantities of interest at the interfaces of the decomposition. As a matter of fact we solve an optimal control problem in which both controls and observation are defined on the interfaces and whose constraints are the PDE's on the overlapping subdomains. 
In this paper we propose and analyze an ICDD method for the Stokes-Darcy coupling, in which the controls are of Dirichlet type: we control the velocity on the interface of the Stokes domain and the pressure on the interface of the Darcy one. The cost functional measures the gap between Stokes and Darcy velocities on one interface and the gap in the pressures on the other one. In this way, both Stokes and Darcy subproblems are closed by very simple (Dirichlet) boundary conditions on the interfaces and there is no need to evaluate neither fluxes nor tangential derivatives, nor to solve auxiliary problems inside the thin layer (overlapping) region. In particular, this feature makes ICDD very suitable to deal with interfaces of arbitrary shape (not only straight lines).

In the case of the Stokes-Darcy coupling the thickness $\delta$ of the overlapping region is crucial to correctly represent the filtration process. It is known that for isotropic media, $\delta$ should be set proportional to the characteristic length of the pores $\varepsilon$ or, after nondimensionalization, to the ratio between $\varepsilon$ and the characteristic length $x_{s}$ of the Stokes domain $[46,40,30]$. Using a totally different argument, a similar quantity $d / h$ was introduced in the asymptotic expansion of Chandesris and Jamet in [8], where $d$ is proportional to the pore dimension, while $h$ is the height of the fluid standing above the porous layer.

At the discrete level the ICDD method uses conforming discretization spaces for both the Stokes velocity and the Darcy pressure, to ensure the correct definition of the optimal control problem.

Numerical comparison with the results obtained using the BJS condition on a sharp interface shows that ICDD provides accurate solutions for both near parallel and near normal flows to the porous media (this terminology follows the classification of Levy and Sanchez-Palencia $[18,35])$. We point out that the numerical comparision with the sharp interface approach and BJS conditions is performed to validate the ICDD method and not to show any equivalence between the two approaches when $\delta \rightarrow 0$. As a matter of fact, there is no reason why ICDD should make sense when $\delta=0$.

The outline of the paper is as follows. In section 2 we present the ICDD method. In section 3 the well-posedness of the optimal control problem in the continuous setting is analyzed. In section 4 we present the $h p$-FEM discretization of the optimal control problem and we focus on its well-posedness and convergence analysis. Finally, section 5 presents several numerical results aimed at corroborating the convergence behavior of the proposed ICDD method and comparing it to the classical coupling strategy relying on the BJS condition on a sharp interface.

2. The ICDD method. Let $\Omega \subset \mathbb{R}^{d}(d=2,3)$ be an open bounded domain with Lipschitz continuous boundary $\partial \Omega$. We consider an overlapping decomposition of $\Omega$ into two subdomains $\Omega_{1}$ and $\Omega_{2}$ such that

$$
\bar{\Omega}=\bar{\Omega}_{1} \cup \bar{\Omega}_{2}, \quad \Omega_{12}=\Omega_{1} \cap \Omega_{2} \neq \emptyset, \quad \Gamma_{i}=\partial \Omega_{i} \backslash \partial \Omega, i=1,2,
$$

as shown in the picture on the left of Figure 2.1. For $i=1,2$, let $\partial \Omega_{i}^{N}$ and $\partial \Omega_{i}^{D}$ be non-empty, non-intersecting, and complementary subsets of $\partial \Omega_{i} \cap \partial \Omega ; \mathbf{n}_{i}$ be the unit normal vector to $\partial \Omega_{i}$ directed outwards the domain $\Omega_{i}$. The following geometrical assumptions will be considered in the whole paper (see the picture on the left of Fig. $2.2)$.

Assumptions 2.1 (Geometric assumptions). For $i=1,2$ (and $j=3-i$ ), let $\partial \Omega_{i}^{D}$ be open in the relative topology of $\partial \Omega_{i}$ with $d_{i}=\operatorname{dist}\left(\overline{\Gamma_{i}}, \overline{\partial \Omega_{i}^{D}}\right)>0$. 

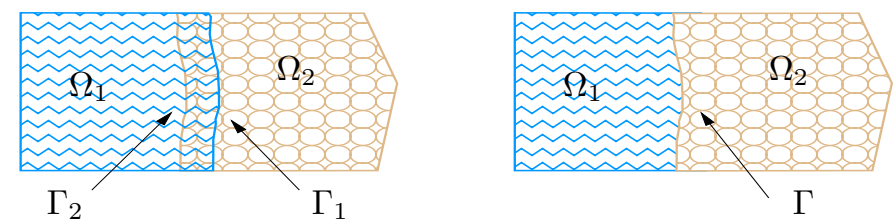

FIG. 2.1. Representation of a $2 D$ section of a possible computational domain for the coupled free/porous-media flow problem. On the left a decomposition with overlap, on the right a decomposition with sharp interface $\Gamma$.
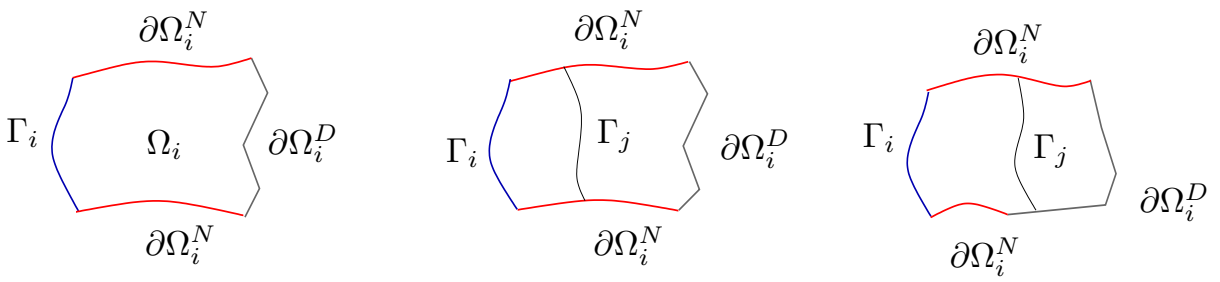

FIG. 2.2. The description of the geometric Assumptions 2.1 (on the left) and 3.2 (on the middle and on the right)

The free/porous-media flow problem is typically modeled by the coupling of Navier-Stokes equations and Darcy law (see, e.g., $[39,17]$ ). However, when the Reynolds number is small the Stokes equations can replace the Navier-Stokes ones, upon neglecting the nonlinear convective term. The same (generalized) Stokes equations are recovered when the time discretization of the Navier-Stokes problem is carried out by using a semi-implicit scheme that treats the convective part explicitly. For these reasons we will present the ICDD method for the Stokes/Darcy problem. With this aim, we introduce two unknown functions $\boldsymbol{\lambda}_{1}$ and $\lambda_{2}$ defined on the interfaces $\Gamma_{1}$ and $\Gamma_{2}$, respectively, that are named controls and play the role of Dirichlet data for the following Stokes and Darcy subproblems, respectively:

Stokes system:

$$
\begin{array}{ll}
-\nabla \cdot \mathbf{T}_{1}\left(\mathbf{u}_{1}, p_{1}\right)=\mathbf{f}_{1} & \text { in } \Omega_{1} \\
\nabla \cdot \mathbf{u}_{1}=0 & \text { in } \Omega_{1} \\
\mathbf{u}_{1}=\boldsymbol{\lambda}_{1} & \text { on } \Gamma_{1} \\
\mathbf{u}_{1}=\mathbf{0} & \text { on } \partial \Omega \\
\mathbf{T}_{1}\left(\mathbf{u}_{1}, p_{1}\right) \mathbf{n}_{1}=\mathbf{0} & \text { on } \partial \Omega
\end{array}
$$

Darcy system:

$$
\begin{array}{ll}
\boldsymbol{\alpha}^{-1} \mathbf{u}_{2}+\nabla p_{2}=\mathbf{f}_{2} & \text { in } \Omega_{2} \\
\nabla \cdot \mathbf{u}_{2}=0 & \text { in } \Omega_{2} \\
p_{2}=\lambda_{2} & \text { on } \Gamma_{2} \\
p_{2}=0 & \text { on } \partial \Omega_{2}^{D} \\
\mathbf{u}_{2} \cdot \mathbf{n}_{2}=0 & \text { on } \partial \Omega_{2}^{N},
\end{array}
$$

where $\mathbf{u}_{1}$ and $p_{1}$ denote, respectively, the velocity and the pressure of the free fluid in $\Omega_{1} ; \mathbf{T}_{1}\left(\mathbf{u}_{1}, p_{1}\right)=\mu\left(\nabla \mathbf{u}_{1}+\left(\nabla \mathbf{u}_{1}\right)^{T}\right)-p_{1} \mathbf{I}$ is the Cauchy stress tensor, where $\mu>0$ is the dynamic viscosity and $\mathbf{u}_{2}$ is the specific discharge (also known as Darcy velocity, seepage velocity, filtration velocity, or volumetric flux density) and represents the average of the fluid velocity over a volume element of the medium (incorporating both solid and fluid material); $p_{2}$ is the intrinsic average pressure of the fluid inside the porous media; $\mathbf{f}_{1}$ and $\mathbf{f}_{2}$ are given external forces and finally $\boldsymbol{\alpha}$ represents a suitable bounded positive definite and symmetric tensor. More precisely, denoting by $g$ the modulus of gravity acceleration, by $z_{0}$ a reference quote, and by $\boldsymbol{\kappa}=\boldsymbol{\kappa}(\mathbf{x})$ the intrinsic permeability of the media, we recover the classical Darcy equations by setting

$$
\boldsymbol{\alpha}=\frac{\boldsymbol{\kappa}}{\mu} \quad \text { and } \quad \mathbf{f}_{2}=-\nabla\left(\rho g\left(z-z_{0}\right)\right)
$$


where $\rho>0$ is the density of the fluid.

The unknown controls $\boldsymbol{\lambda}_{1}$ and $\lambda_{2}$ in (2.1) can be obtained by minimizing a suitable cost functional that measures the gap between the velocities and the pressures on the interfaces $\Gamma_{1}$ and $\Gamma_{2}$, respectively, that is

$$
\inf _{\left(\boldsymbol{\lambda}_{1}, \lambda_{2}\right)}\left[J\left(\boldsymbol{\lambda}_{1}, \lambda_{2}\right)=\frac{1}{2}\left\|\mathbf{u}_{1}\left(\boldsymbol{\lambda}_{1}\right)-\mathbf{u}_{2}\left(\lambda_{2}\right)\right\|_{\Gamma_{1}}^{2}+\frac{1}{2}\left\|p_{2}\left(\lambda_{2}\right)-p_{1}\left(\boldsymbol{\lambda}_{1}\right)\right\|_{\Gamma_{2}}^{2}\right],
$$

in suitable norms. For the time being, this expression is a formal one. We will better specify the meaning of $J$ after introducing the functional spaces.

Problem (2.1)-(2.2) is in fact an optimal control problem, in which $\left(\mathbf{u}_{i}, p_{i}\right)$ (for $i=1,2)$ represent the state variables. The solutions of $(2.1)-(2.2)$ depend on both the controls, the right hand side of the momentum equation and the boundary data, but we omit at this stage such dependences for sake of notation.

3. Analysis of ICDD. In this section we analyze the problem in the continuous setting. First we study the well-posedness and the regularity for the subproblems, then we apply such results to prove that the optimal control problem is well defined.

3.1. Weak formulation of the subproblems and regularity results. We introduce the functional spaces

$$
\begin{aligned}
& H(\operatorname{div}, \Omega)=\left\{\mathbf{v} \in\left[L^{2}(\Omega)\right]^{d}: \nabla \cdot \mathbf{v} \in L^{2}(\Omega)\right\}, \\
& \mathbf{V}_{1}=\left\{\mathbf{v}_{1} \in\left[H^{1}\left(\Omega_{1}\right)\right]^{d}:\left.\mathbf{v}_{1}\right|_{\partial \Omega_{1}^{D}}=\mathbf{0}\right\}, \mathbf{V}_{1}^{0}=\left\{\mathbf{v}_{1} \in \mathbf{V}_{1}:\left.\mathbf{v}_{1}\right|_{\Gamma_{1}}=\mathbf{0}\right\}, Q_{1}=L^{2}\left(\Omega_{1}\right), \\
& \mathbf{V}_{2}=\left\{\mathbf{v}_{2} \in H\left(\operatorname{div}, \Omega_{2}\right): \mathbf{v}_{2} \cdot \mathbf{n}_{2}=0 \text { on } \partial \Omega_{2}^{N}\right\}, \quad Q_{2}=L^{2}\left(\Omega_{2}\right), \\
& \mathbf{\Lambda}_{1}=\left[H^{1 / 2}\left(\Gamma_{1}\right)\right]^{d}, \quad \Lambda_{2}=H^{1 / 2}\left(\Gamma_{2}\right) .
\end{aligned}
$$

Assumptions 3.1 (Data assumptions). We assume that $\mathbf{f}_{1} \in\left[L^{2}\left(\Omega_{1}\right)\right]^{d}, \mathbf{f}_{2} \in$ $H\left(\right.$ div,$\left.\Omega_{2}\right)$, and $\exists \lambda_{\alpha}>0$ such that the symmetric tensor $\boldsymbol{\alpha} \in\left[L^{\infty}\left(\Omega_{2}\right)\right]^{d \times d}$ satisfies $\xi^{T} \boldsymbol{\alpha} \xi \geq \lambda_{\alpha}|\xi|^{2}, \forall \xi \in \mathbb{R}^{d}$.

Should $\boldsymbol{\lambda}_{1} \in \boldsymbol{\Lambda}_{1}, \lambda_{2} \in \Lambda_{2}$ be known, the weak form of system (2.1) reads: given $\mathbf{f}_{1} \in\left[L^{2}\left(\Omega_{1}\right)\right]^{d}, \mathbf{f}_{2} \in H\left(\operatorname{div}, \Omega_{2}\right)$, look for $\left(\mathbf{u}_{1}, p_{1}\right) \in \mathbf{V}_{1} \times Q_{1}$ with $\mathbf{u}_{1}=\boldsymbol{\lambda}_{1}$ on $\Gamma_{1}$ and $\left(\mathbf{u}_{2}, p_{2}\right) \in \mathbf{V}_{2} \times Q_{2}$ s.t.

$$
\begin{array}{ll}
a_{1}\left(\mathbf{u}_{1}, \mathbf{v}_{1}\right)+b_{1}\left(\mathbf{v}_{1}, p_{1}\right)=\mathcal{F}_{1}\left(\mathbf{v}_{1}\right) & \forall \mathbf{v}_{1} \in \mathbf{V}_{1}^{0} \\
b_{1}\left(\mathbf{u}_{1}, q_{1}\right)=0 & \forall q_{1} \in Q_{1}
\end{array}
$$

and

$$
\begin{array}{ll}
a_{2}\left(\mathbf{u}_{2}, \mathbf{v}_{2}\right)+b_{2}\left(\mathbf{v}_{2}, p_{2}\right)=\mathcal{F}_{2}\left(\mathbf{v}_{2}\right)-\left\langle\mathbf{v}_{2} \cdot \mathbf{n}_{2}, \lambda_{2}\right\rangle_{\Gamma_{2}} & \forall \mathbf{v}_{2} \in \mathbf{V}_{2} \\
b_{2}\left(\mathbf{u}_{2}, q_{2}\right)=0 & \forall q_{2} \in Q_{2},
\end{array}
$$

where:

$$
\begin{array}{ll}
a_{1}: \mathbf{V}_{1} \times \mathbf{V}_{1} \rightarrow \mathbb{R}, & a_{1}\left(\mathbf{u}_{1}, \mathbf{v}_{1}\right)=\int_{\Omega_{1}} \frac{\mu}{2}\left(\nabla \mathbf{u}_{1}+\left(\nabla \mathbf{u}_{1}\right)^{T}\right):\left(\nabla \mathbf{v}_{1}+\left(\nabla \mathbf{v}_{1}\right)^{T}\right) d \Omega, \\
a_{2}: \mathbf{V}_{2} \times \mathbf{V}_{2} \rightarrow \mathbb{R}, & a_{2}\left(\mathbf{u}_{2}, \mathbf{v}_{2}\right)=\int_{\Omega_{2}}\left(\boldsymbol{\alpha}^{-1} \mathbf{u}_{2}\right) \cdot \mathbf{v}_{2} d \Omega \\
b_{i}: \mathbf{V}_{i} \times Q_{i} \rightarrow \mathbb{R}, & b_{i}\left(\mathbf{v}_{i}, q_{i}\right)=-\int_{\Omega_{i}} q_{i} \nabla \cdot \mathbf{v}_{i} d \Omega, \\
\mathcal{F}_{i}: \mathbf{V}_{i} \rightarrow \mathbb{R}, & \mathcal{F}_{i}\left(\mathbf{v}_{i}\right)=\int_{\Omega_{i}} \mathbf{f}_{i} \cdot \mathbf{v}_{i} d \Omega, \quad i=1,2,
\end{array}
$$


while $\langle\cdot, \cdot\rangle_{\Gamma_{2}}$ denotes the duality pairing between $H^{1 / 2}\left(\Gamma_{2}\right)$ and its dual space. The inner product and the norm in $H^{1 / 2}\left(\Gamma_{i}\right)$ will be denoted by $(\cdot, \cdot)_{\frac{1}{2}, \Gamma_{i}}$ and $\|\cdot\|_{\frac{1}{2}, \Gamma_{i}}$, respectively. The following well-posedness result holds true.

TheOREM 3.1 (Well posedness). Let Assumptions 2.1 and 3.1 be satisfied. If $\boldsymbol{\lambda}_{1} \in \boldsymbol{\Lambda}_{1}$, and $\lambda_{2} \in \Lambda_{2}$, then both problems (3.1) and (3.2) are well-posed, with in addition $p_{2} \in H^{1}\left(\Omega_{2}\right)$. Moreover

$$
\begin{aligned}
& \left\|\mathbf{u}_{1}\right\|_{V_{1}} \leq C_{1}\left(\frac{\left\|\mathbf{f}_{1}\right\|_{L^{2}\left(\Omega_{1}\right)}}{\mu}+\left\|\boldsymbol{\lambda}_{1}\right\|_{\frac{1}{2}, \Gamma_{1}}\right) \\
& \left\|p_{1}\right\|_{L^{2}\left(\Omega_{1}\right)} \leq C_{1}\left(\left\|\mathbf{f}_{1}\right\|_{L^{2}\left(\Omega_{1}\right)}+\mu\left\|\boldsymbol{\lambda}_{1}\right\|_{\frac{1}{2}, \Gamma_{1}}\right)
\end{aligned}
$$

and

$$
\left\|p_{2}\right\|_{H^{1}\left(\Omega_{2}\right)} \leq C_{2}\left(\left\|\mathbf{f}_{2}\right\|_{L^{2}\left(\Omega_{2}\right)}+\left\|\lambda_{2}\right\|_{\frac{1}{2}, \Gamma_{2}}\right),
$$

for suitable constants $C_{1}=C_{1}\left(\Omega_{1}, \partial \Omega_{1}^{D}, \Gamma_{1}\right)$ and $C_{2}=C_{2}\left(\Omega_{2}, \partial \Omega_{2}^{D}, \Gamma_{2}, \boldsymbol{\alpha}\right)$.

Proof. Let us consider the Stokes problem. The velocity field solution of (3.1) can be written as $\mathbf{u}_{1}=\mathbf{u}_{1,0}+E_{1} \boldsymbol{\lambda}_{1}$, where $\left(\mathbf{u}_{1,0}, p_{1}\right) \in \mathbf{V}_{1}^{0} \times Q_{1}$ is the solution of

$$
\begin{array}{ll}
a_{1}\left(\mathbf{u}_{1,0}, \mathbf{v}_{1}\right)+b_{1}\left(\mathbf{v}_{1}, p_{1}\right)=\left(\mathbf{f}_{1}, \mathbf{v}_{1}\right)_{L^{2}(\Omega)}-a_{1}\left(E_{1} \boldsymbol{\lambda}_{1}, \mathbf{v}_{1}\right) & \forall \mathbf{v}_{1} \in \mathbf{V}_{1}^{0} \\
b_{1}\left(\mathbf{u}_{1,0}, q_{1}\right)=-b_{1}\left(E_{1} \boldsymbol{\lambda}_{1}, q_{1}\right) & \forall q_{1} \in Q_{1},
\end{array}
$$

and $E_{1} \boldsymbol{\lambda}_{1} \in \mathbf{V}_{1}$ is an extension of $\boldsymbol{\lambda}_{1}$. We claim that we can assume

$$
\left\|\nabla\left(E_{1} \boldsymbol{\lambda}_{1}\right)\right\|_{L^{2}\left(\Omega_{1}\right)} \leq c_{1}\left\|\boldsymbol{\lambda}_{1}\right\|_{\frac{1}{2}, \Gamma_{1}}
$$

where $c_{1}$ depends on the triplet $\left(\Omega_{1}, \partial \Omega_{1}^{D}, \Gamma_{1}\right)$. The bilinear form $a_{1}$ is continuous and coercive on $\|\cdot\|_{V_{1}}$, coercivity being a consequence of Korn's inequality (see e.g. $[44,(5.2 .5)])$

$$
\int_{\Omega_{1}}\left(\nabla \mathbf{v}+(\nabla \mathbf{v})^{T}\right):\left(\nabla \mathbf{v}+(\nabla \mathbf{v})^{T}\right) \geq K^{*}\left(\Omega_{1}, \partial \Omega_{1}^{D}\right)\|\mathbf{v}\|_{V_{1}}
$$

for any $\mathbf{v} \in \mathbf{V}_{1}^{0}$. The bilinear form $b_{1}$ is continuous and satisfies the inf-sup condition [44, Prop. 5.3.2]. By applying classical results on saddle-point problems ([2, Thm. 4.2.1]), (3.6) admits a unique solution. Moreover, estimate (3.4) is a consequence of [2, Cor. 4.2.1], and of (3.7).

We achieve estimates (3.7) by choosing $E_{1} \boldsymbol{\lambda}_{1} \in \mathbf{V}_{1}$ as a harmonic extension with Neumann conditions on $\partial \Omega_{1}^{N}$, i.e., such that

$$
\left(\nabla E_{1} \boldsymbol{\lambda}_{1}, \nabla \mathbf{v}_{1}\right)_{L^{2}\left(\Omega_{1}\right)}=0 \quad \forall \mathbf{v}_{1} \in \mathbf{V}_{1}^{0} .
$$

This can be proved as follows. On $\boldsymbol{\Lambda}_{1}$ we define the norm

$$
\left\|\boldsymbol{\lambda}_{1}\right\|_{*}=\inf \left\{\|\mathbf{v}\|_{H^{1}\left(\Omega_{1}\right)}: \mathbf{v}=\boldsymbol{\lambda}_{1} \text { on } \Gamma_{1}, \mathbf{v}=\mathbf{0} \text { on } \partial \Omega_{1}^{D}\right\} .
$$

In view of the geometrical assumptions $2.1,\|\cdot\|_{*}$ is equivalent to the canonical norm in $H^{1 / 2}\left(\Gamma_{1}\right)$. As a matter of fact

$$
\begin{aligned}
\left\|\boldsymbol{\lambda}_{1}\right\|_{\frac{1}{2}, \Gamma_{2}} & =\inf \left\{\|\mathbf{v}\|_{H^{1}\left(\Omega_{1}\right)}: \mathbf{v}=\boldsymbol{\lambda}_{1} \text { on } \Gamma_{1}\right\} \\
& \leq \inf \left\{\|\mathbf{v}\|_{H^{1}\left(\Omega_{1}\right)}: \mathbf{v}=\boldsymbol{\lambda}_{1} \text { on } \Gamma_{1}, \mathbf{v}=\mathbf{0} \text { on } \partial \Omega_{1}^{D}\right\}=\left\|\boldsymbol{\lambda}_{1}\right\|_{*} .
\end{aligned}
$$


Vice versa, being $d_{1}=\operatorname{dist}\left(\overline{\Gamma_{1}}, \overline{\partial \Omega_{1}^{D}}\right)>0$, there exist functions $\varphi \in C_{c}^{\infty}\left(\mathbb{R}^{d}\right)$ s.t. $\varphi=1 \mathrm{in}$ an open set containing $\overline{\Gamma_{1}}$ and $\varphi=0$ in an open set containing $\overline{\partial \Omega_{1}^{D}}$. Let $\mathbf{v} \in H^{1}\left(\Omega_{1}\right)$ s.t. $\mathbf{v}_{\mid \Gamma_{1}}=\boldsymbol{\lambda}_{1}$, then $(\varphi \mathbf{v}) \in H^{1}\left(\Omega_{1}\right),(\varphi \mathbf{v})_{\mid \Gamma_{1}}=\boldsymbol{\lambda}_{1},(\varphi \mathbf{v})_{\mid \partial \Omega_{1}^{D}}=0$, and $\left\|\boldsymbol{\lambda}_{1}\right\|_{*} \leq\|\varphi \mathbf{v}\|_{H^{1}\left(\Omega_{1}\right)} \leq C_{\varphi}\|\mathbf{v}\|_{H^{1}\left(\Omega_{1}\right)}$. By taking the infimum over $\mathbf{v}$, it holds

$$
\left\|\boldsymbol{\lambda}_{1}\right\|_{*} \leq C_{\varphi} \inf \left\{\|\mathbf{v}\|_{H^{1}\left(\Omega_{1}\right)}: \mathbf{v}=\boldsymbol{\lambda}_{1} \text { on } \Gamma_{1}\right\}=C_{\varphi}\left\|\boldsymbol{\lambda}_{1}\right\|_{\frac{1}{2}, \Gamma_{1}} .
$$

Since clearly $\left\|\nabla\left(E_{1} \boldsymbol{\lambda}_{1}\right)\right\|_{L^{2}\left(\Omega_{1}\right)} \leq\left\|\boldsymbol{\lambda}_{1}\right\|_{*}$, claim (3.7) follows (note that $C_{\varphi}$ depends only on the geometry of $\left.\left(\Omega_{1}, \partial \Omega_{1}^{D}, \Gamma_{1}\right)\right)$.

Existence of a unique solution for the Darcy problem can be obtained in a similar way. (See [2, Sect. 4.2.5] for the existence, and [43, Thm 7.1.2] for the higher regularity of $p_{2}$. See also Remark 3.2 below). Estimate (3.5) is a consequence of classical results on elliptic problems (together with the scalar analogue of (3.7)).

REMARK 3.1. In view of the proof above, the constant $C_{1}$ appearing in (3.4) depends on the geometry of the triplet $\left(\Omega_{1}, \partial \Omega_{1}^{D}, \Gamma_{1}\right)$. In particular the constant $C_{1}$ increases as $d_{1}=\operatorname{dist}\left(\overline{\Gamma_{1}}, \overline{\partial \Omega_{1}^{D}}\right)$ decreases. Roughly speaking, we can assume $C_{1} \sim$ $d_{1}^{-1}$. The dependence of $C_{2}$ in (3.5) upon both $\boldsymbol{\alpha}$ and $\left(\Omega_{2}, \partial \Omega_{2}^{D}, \Gamma_{2}\right)$ is readily seen (see Remark 3.2 below) to be of the form

$$
C_{2}=\sqrt{\frac{\|\boldsymbol{\alpha}\|_{\infty}}{\lambda_{\alpha}}} \sqrt{1+\frac{\|\boldsymbol{\alpha}\|_{\infty}}{\lambda_{\alpha}}} \hat{C}_{2}\left(\Omega_{2}, \partial \Omega_{2}^{D}, \Gamma_{2}\right)
$$

where $\lambda_{\alpha}$ is the coercivity constant of $\boldsymbol{\alpha}$, and the dependence of $\hat{C}_{2}$ on the triplet $\left(\Omega_{2}, \partial \Omega_{2}^{D}, \Gamma_{2}\right)$ having the same features already seen for $C_{1}$.

REMARK 3.2. The Darcy problem can be reformulated as an elliptic problem for the pressure $p_{2}$ as follows, making both the higher regularity for the pressure and the associated estimate more transparent. As a matter of fact $p_{2} \in H^{1}\left(\Omega_{2}\right)$ is such that for every $\varphi \in H^{1}\left(\Omega_{2}\right)$ with $\varphi=0$ on $\Gamma_{2} \cup \partial \Omega_{2}^{D}$

$$
\int_{\Omega_{2}} \boldsymbol{\alpha} \nabla p_{2} \cdot \nabla \varphi d \Omega=\int_{\Omega_{2}} \boldsymbol{\alpha} \boldsymbol{f}_{2} \cdot \nabla \varphi d \Omega
$$

The associated velocity $\boldsymbol{u}_{2}$ is recovered by setting $\boldsymbol{u}_{2}=\alpha\left(\boldsymbol{f}_{2}-\nabla p_{2}\right)$.

The following theorem establishes the local regularity of the solutions of (3.1) and (3.2).

Theorem 3.2 (Regularity). Let Assumptions 2.1 and 3.1 be satisfied. For $i=$ 1,2 , let $\Omega^{\prime} \subseteq \Omega_{i}$ be such that $\overline{\Omega^{\prime}} \cap \partial \Omega_{i} \subset A_{i}$, with $A_{i} \subset\left(\partial \Omega_{i}^{D} \cup \operatorname{int}\left(\partial \Omega_{i}^{N}\right)\right)$ open and of class $C^{2,1}$ (openness and internal part are intended in the relative topology of the boundary). Then the following items hold true.

1. Let $\left(\mathbf{u}_{1}, p_{1}\right) \in \mathbf{V}_{1} \times Q_{1}$ be the solution of (3.1). Then $\mathbf{u}_{1} \in H^{2}\left(\Omega^{\prime}\right), p_{1} \in$ $H^{1}\left(\Omega^{\prime}\right)$ and there exists a positive constant $C_{3}\left(\Omega^{\prime}\right)$ s.t.

$$
\mu\left\|\mathbf{u}_{1}\right\|_{H^{2}\left(\Omega^{\prime}\right)}+\left\|p_{1}\right\|_{H^{1}\left(\Omega^{\prime}\right)} \leq C_{3}\left(\left\|\mathbf{f}_{1}\right\|_{L^{2}\left(\Omega_{1}\right)}+\mu\left\|\mathbf{u}_{1}\right\|_{H^{1}\left(\Omega_{1}\right)}+\left\|p_{1}\right\|_{L^{2}\left(\Omega_{1}\right)}\right) .
$$

2. Let $\left(\mathbf{u}_{2}, p_{2}\right) \in \mathbf{V}_{2} \times Q_{2}$ be the solution of (3.2). If $\boldsymbol{\alpha}$ is of class $C^{1}$ on $\bar{\Omega}_{2}$ and $\boldsymbol{f}_{2} \in H\left(\right.$ div,$\left.\Omega_{2}\right) \cap H^{1}\left(\Omega_{2}\right)$, then $\mathbf{u}_{2} \in H^{1}\left(\Omega^{\prime}\right), p_{2} \in H^{2}\left(\Omega^{\prime}\right)$ and there exists a positive constant $C_{4}\left(\Omega^{\prime},\|\boldsymbol{\alpha}\|_{C^{1}}\right)$ s.t.

$$
\left\|\mathbf{u}_{2}\right\|_{H^{1}\left(\Omega^{\prime}\right)}+\left\|p_{2}\right\|_{H^{2}\left(\Omega^{\prime}\right)} \leq C_{4}\left(\left\|\mathbf{f}_{2}\right\|_{H^{1}\left(\Omega_{2}\right)}+\left\|p_{2}\right\|_{H^{1}\left(\Omega_{2}\right)}\right) .
$$


Proof. Concerning the Stokes problem, interior regularity and regularity up to the Dirichlet part of the boundary are contained in Chapter 3 of [10] (Lemma 3.6 and Theorem 3.7). The extension to the regularity up to the Neumann part of the boundary can be achieved by adapting the same arguments. More precisely: regular cut-off functions are used to localize the problem near the Neumann part of the boundary; the change of coordinates is employed to flatten the boundary (this is the reason why we require that $A_{i}$ is $C^{2,1}$ regular); Nieremberg method is used to bound the incremental quotients in tangential direction for both $\nabla \mathbf{u}_{1}$ and $p_{1}$; information concerning normal directions are inferred using the Stokes equations, hence the full local $H^{2}$ regularity for the velocity and full local $H^{1}$ regularity for the pressure, together with the associated estimate (3.10) through a covering argument.

Concerning the Darcy problem, we employ the equivalent reformulation detailed in Remark 3.2. Then as a consequence of standard elliptic regularity (see, e.g., [22]), we obtain that $p_{2} \in H^{2}\left(\Omega^{\prime}\right)$, hence also $\mathbf{u}_{2} \in H^{1}\left(\Omega^{\prime}\right)$ in view of $\mathbf{u}_{2}=\boldsymbol{\alpha}\left(\mathbf{f}_{2}-\nabla p_{2}\right)$, together with estimate (3.11) through a covering argument.

REMARK 3.3. Notice that the constant $C_{3}\left(\Omega^{\prime}\right)$ increases as the distance between $\Omega^{\prime}$ and $\overline{\Gamma_{1}} \cup\left(\overline{\partial \Omega_{1}^{D}} \cap \partial \Omega_{1}^{N}\right)$ decreases: roughly speaking, we can say that it is proportional to the inverse of this distance. The same can be said for $C_{4}\left(\Omega^{\prime},\|\boldsymbol{\alpha}\|_{C^{1}}\right)$.

The previous result motivates the following assumptions (see also Fig. 2.2).

Assumptions 3.2 (Regularity assumptions). For $i=1,2$ (and $j=3-i$ ), let $\overline{\Gamma_{j}} \cap \partial \Omega_{i} \subset A_{i}$, with $A_{i} \subset\left(\partial \Omega_{i}^{D} \cup \operatorname{int}\left(\partial \Omega_{i}^{N}\right)\right)$ open and of class $C^{2,1}$ (openness and internal part are intended in the relative topology of the boundary). Moreover, let $\boldsymbol{\alpha} \in\left[C^{1}\left(\bar{\Omega}_{2}\right)\right]^{d \times d}$ and $\mathbf{f}_{2} \in H\left(\operatorname{div}, \Omega_{2}\right) \cap H^{1}\left(\Omega_{2}\right)$.

Notice that Assumptions 3.2 entails that $\overline{\Gamma_{j}}$ is far enough from the points of the boundary where boundary conditions change from Dirichlet to Neumann and that

$$
\delta=\operatorname{dist}\left(\Gamma_{1}, \Gamma_{2}\right)>0 .
$$

We can think of $\delta$ as the characteristic length of the overlapping between $\Omega_{1}$ and $\Omega_{2}$.

REMARK 3.4. Assumptions 3.2 imply that the traces of $p_{1}$ on $\Gamma_{2}$ and $\mathbf{u}_{2}$ on $\Gamma_{1}$ are well-defined as elements in $H^{1 / 2}$ (we can choose $\Omega^{\prime}$ containing $\Gamma_{i}$ ), so that we can characterize the norms involved in the minimization problem (2.2) by choosing $H^{1 / 2}\left(\Gamma_{i}\right)$ norms.

3.2. Well-posedness of the optimal control problem. Let us formally write the solutions $\left(\mathbf{u}_{i}, p_{i}\right)$ of (3.1) and (3.2) as $\mathbf{u}_{i}=\mathbf{u}_{i}^{\boldsymbol{\lambda}_{i}, \mathbf{f}_{i}}$ and $p_{i}=p_{i}^{\boldsymbol{\lambda}_{i}, \mathbf{f}_{i}}$. Upon setting $\boldsymbol{\Lambda}=\boldsymbol{\Lambda}_{1} \times \Lambda_{2}$, the ICDD method to solve the Stokes-Darcy problem (2.1) reads as a constrained minimization problem: look for

$$
\inf _{\boldsymbol{\lambda} \in \boldsymbol{\Lambda}}\left[J(\boldsymbol{\lambda})=\frac{1}{2}\left\|\mathbf{u}_{1}^{\boldsymbol{\lambda}_{1}, \mathbf{f}_{1}}-\mathbf{u}_{2}^{\lambda_{2}, \mathbf{f}_{2}}\right\|_{\frac{1}{2}, \Gamma_{1}}^{2}+\frac{1}{2}\left\|p_{2}^{\lambda_{2}, \mathbf{f}_{2}}-p_{1}^{\boldsymbol{\lambda}_{1}, \mathbf{f}_{1}}\right\|_{\frac{1}{2}, \Gamma_{2}}^{2}\right] .
$$

The main result of this section is that under suitable assumptions on the geometry of the domains and on the coefficients, problem (3.13) admits a unique solution.

Let us set

$$
\mathbf{u}_{i}^{\boldsymbol{\lambda}_{i}}=\mathbf{u}_{i}^{\boldsymbol{\lambda}_{i}, \mathbf{0}} \quad \text { and } \quad p_{i}^{\boldsymbol{\lambda}_{i}}=p_{i}^{\boldsymbol{\lambda}_{i}, \mathbf{0}} .
$$

The following lemma provides stability estimates at the interfaces which will be useful in the sequel.

Lemma 3.3. Let Assumptions 2.1, 3.1, and 3.2 be satisfied. Then

$$
\left\|p_{1 \mid \Gamma_{2}}^{\boldsymbol{\lambda}_{1}}\right\|_{\frac{1}{2}, \Gamma_{2}} \leq \mu C_{1} C_{3}\left\|\boldsymbol{\lambda}_{1}\right\|_{\frac{1}{2}, \Gamma_{1}}
$$


and

$$
\left\|\mathbf{u}_{2}^{\lambda_{2}}{ }_{\mid \Gamma_{1}}\right\|_{\frac{1}{2}, \Gamma_{1}} \leq\|\boldsymbol{\alpha}\|_{C^{1}} C_{2} C_{4}\left\|\lambda_{2}\right\|_{\frac{1}{2}, \Gamma_{2}}
$$

where $C_{1}, C_{2}$ are defined in Theorem 3.1, while $C_{3}, C_{4}$ are defined in Theorem 3.2.

Proof. In view of Assumptions 3.2 we can apply Lemma 3.2 to $\left(\mathbf{u}_{1}^{\boldsymbol{\lambda}_{1}}, p_{1}^{\boldsymbol{\lambda}_{1}}\right)$, by choosing an admissible $\Omega^{\prime}$ s.t. $\Gamma_{2} \subset \Omega^{\prime}$. Estimate (3.14) follows then combining (3.10) and (3.4). The proof of estimate (3.15) is similar recalling that $\mathbf{u}_{2}^{\lambda_{2}}=-\boldsymbol{\alpha} \nabla p_{2}^{\lambda_{2}}$.

For any $\boldsymbol{\lambda}=\left(\boldsymbol{\lambda}_{1}, \lambda_{2}\right) \in \boldsymbol{\Lambda}=\boldsymbol{\Lambda}_{1} \times \Lambda_{2}$, we define

$$
\|\boldsymbol{\lambda}\|=\left(\left\|\mathbf{u}_{1}^{\boldsymbol{\lambda}_{1}}-\mathbf{u}_{2}^{\lambda_{2}}\right\|_{\frac{1}{2}, \Gamma_{1}}^{2}+\left\|p_{2}^{\lambda_{2}}-p_{1}^{\boldsymbol{\lambda}_{1}}\right\|_{\frac{1}{2}, \Gamma_{2}}^{2}\right)^{1 / 2} .
$$

Lemma 3.4. Let Assumptions 2.1, 3.1, and 3.2 be satisfied. Let

$$
\mu\|\boldsymbol{\alpha}\|_{C^{1}} C_{1} C_{2} C_{3} C_{4}<1
$$

where $C_{1}, C_{2}$ are defined in Theorem 3.1, while $C_{3}, C_{4}$ are defined in Theorem 3.2. Then $\|\cdot\|$ is a norm on the control space $\boldsymbol{\Lambda}$ equivalent to the canonical norm

$$
\|\boldsymbol{\lambda}\|_{\boldsymbol{\Lambda}}=\left(\left\|\boldsymbol{\lambda}_{1}\right\|_{\frac{1}{2}, \Gamma_{1}}^{2}+\left\|\lambda_{2}\right\|_{\frac{1}{2}, \Gamma_{2}}^{2}\right)^{1 / 2}
$$

Proof. $\||\| \cdot| \mid$ is obviously a seminorm, therefore we limit ourselves to show that $\|\boldsymbol{\lambda}\|=0$ implies $\boldsymbol{\lambda}=\mathbf{0}$. We observe that $\|\boldsymbol{\lambda}\|=0$ implies

$$
\boldsymbol{\lambda}_{1}=\mathbf{u}_{2}^{\lambda_{2}} \Gamma_{1}, \quad \lambda_{2}=p_{1}^{\boldsymbol{\lambda}_{1}} \Gamma_{2} .
$$

Then, we have to prove that the unique solution of (3.1), (3.2), (3.18) is $\boldsymbol{\lambda}=$ $\left(\boldsymbol{\lambda}_{1}, \lambda_{2}\right)=(\mathbf{0}, 0)$. We define the linear map $\psi: \boldsymbol{\Lambda} \rightarrow \boldsymbol{\Lambda}$ such that

$$
\boldsymbol{\lambda}=\left[\begin{array}{c}
\boldsymbol{\lambda}_{1} \\
\lambda_{2}
\end{array}\right] \mapsto\left[\begin{array}{c}
\left(\mathbf{u}_{1}^{\boldsymbol{\lambda}_{1}}, p_{1}^{\boldsymbol{\lambda}_{1}}\right) \\
\left(\mathbf{u}_{2}^{\lambda_{2}}, p_{2}^{\lambda_{2}}\right)
\end{array}\right] \mapsto\left[\begin{array}{c}
\mathbf{u}_{2}^{\lambda_{2}} \mid \Gamma_{1} \\
p_{1}^{\boldsymbol{\lambda}_{1}} \mid \Gamma_{2}
\end{array}\right]=\psi(\boldsymbol{\lambda})
$$

We observe that $\boldsymbol{\lambda}$ is a solution of (3.1), (3.2), (3.18) if and only if $\psi(\boldsymbol{\lambda})=\boldsymbol{\lambda}$.

If $\psi(\boldsymbol{\lambda})=\boldsymbol{\lambda}$, then in view of (3.14) and (3.15) we infer

$$
\left\|\boldsymbol{\lambda}_{1}\right\|_{\frac{1}{2}, \Gamma_{1}} \leq\|\boldsymbol{\alpha}\|_{C^{1}} C_{2} C_{4}\left\|\lambda_{2}\right\|_{\frac{1}{2}, \Gamma_{2}} \quad \text { and } \quad\left\|\lambda_{2}\right\|_{\frac{1}{2}, \Gamma_{2}} \leq \mu C_{1} C_{3}\left\|\boldsymbol{\lambda}_{1}\right\|_{\frac{1}{2}, \Gamma_{1}},
$$

and thus

$$
\left\|\lambda_{2}\right\|_{\frac{1}{2}, \Gamma_{2}} \leq \mu\|\boldsymbol{\alpha}\|_{C^{1}} C_{1} C_{2} C_{3} C_{4}\left\|\lambda_{2}\right\|_{\frac{1}{2}, \Gamma_{2}} .
$$

In view of (3.17) we infer $\lambda_{2}=0$. Similar calculations show that also $\boldsymbol{\lambda}_{1}=0$.

Inequality (3.17) entails also the equivalence of the two norms. In view of Lemma 3.3 the norm $\|\cdot\|$ is controlled by $\|\cdot\|_{\Lambda}$. Vice versa, we have

$$
\begin{aligned}
\left\|\boldsymbol{\lambda}_{1}\right\|_{\frac{1}{2}, \Gamma_{1}} & \leq\left\|\mathbf{u}_{1}^{\boldsymbol{\lambda}_{1}}-\mathbf{u}_{2}^{\lambda_{2}}\right\|_{\frac{1}{2}, \Gamma_{1}}+\|\boldsymbol{\alpha}\|_{C^{1}} C_{2} C_{4}\left\|\lambda_{2}\right\|_{\frac{1}{2}, \Gamma_{2}}, \\
\left\|\lambda_{2}\right\|_{\frac{1}{2}, \Gamma_{2}} & \leq\left\|p_{2}^{\lambda_{2}}-p_{1}^{\boldsymbol{\lambda}_{1}}\right\|_{\frac{1}{2}, \Gamma_{2}}+\mu C_{1} C_{3}\left\|\boldsymbol{\lambda}_{1}\right\|_{\frac{1}{2}, \Gamma_{1}},
\end{aligned}
$$

so that

$$
\left(1-\mu\|\boldsymbol{\alpha}\|_{C^{1}} C_{1} C_{2} C_{3} C_{4}\right)\left\|\boldsymbol{\lambda}_{1}\right\|_{\frac{1}{2}, \Gamma_{1}} \leq\left\|\mathbf{u}_{1}^{\boldsymbol{\lambda}_{1}}-\mathbf{u}_{2}^{\lambda_{2}}\right\|_{\frac{1}{2}, \Gamma_{1}}+\|\boldsymbol{\alpha}\|_{C^{1}} C_{2} C_{4}\left\|p_{2}^{\lambda_{2}}-p_{1}^{\boldsymbol{\lambda}_{1}}\right\|_{\frac{1}{2}, \Gamma_{2}}
$$


and a similar estimate holds for $\left\|\lambda_{2}\right\|_{\frac{1}{2}, \Gamma_{2}}$. The equivalence between the two norms immediately follows.

REMARK 3.5. Fixing the geometry, condition (3.17) can be achieved by taking $\mu$ or $\|\boldsymbol{\alpha}\|_{C^{1}}$ (with $\|\boldsymbol{\alpha}\|_{\infty} / \lambda_{\boldsymbol{\alpha}}$ bounded from above) sufficiently small. In the isotropic case

$$
\boldsymbol{\alpha}=\frac{\kappa}{\mu} \mathbf{I}
$$

and in the case in which the thickness overlap $\delta$ (defined in (3.12)) is small, so that $C_{3}$ and $C_{4}$ behave essentially as $\delta^{-1}$, in view of Remarks 3.1 and 3.3 we get the condition $\kappa C(\delta)<1$, where $C(\delta) \sim \delta^{-2}$. This is in agreement with the requirement $\delta \sim \sqrt{\kappa} \sim \varepsilon$ suggested by the physical analysis of the filtration of fluids in porous media (being $\varepsilon$ the porous size). See the comment in the introduction and Sect. 5.

REMARK 3.6. The requirement $d_{i}=\operatorname{dist}\left(\overline{\Gamma_{i}}, \overline{\partial \Omega_{i}^{D}}\right)>0$ for $i=1,2$ entailed by Assumptions 2.1 is crucial for our arguments in the proof of Lemma 3.4. If indeed $d_{i}=0$, then estimates (3.4) and (3.5) would involve the $H_{00}^{1 / 2}$ norms of $\boldsymbol{\lambda}_{1}$ and $\lambda_{2}$. However, the traces of $\mathbf{u}_{2}^{\lambda_{2}}$ on $\Gamma_{1}$ and of $p_{1}^{\boldsymbol{\lambda}_{1}}$ on $\Gamma_{2}$ do not belong to $H_{00}^{1 / 2}$ in general, so that the form of (3.16) should remain unchanged. Since the $H^{1 / 2}$ norm is strictly weaker than that of $H_{00}^{1 / 2}$, the arguments employed above fail.

Let us define the bilinear form $\pi: \boldsymbol{\Lambda} \times \boldsymbol{\Lambda} \rightarrow \mathbb{R}$

$$
\pi(\boldsymbol{\lambda}, \boldsymbol{\eta})=\frac{1}{2}\left(\mathbf{u}_{1}^{\boldsymbol{\lambda}_{1}}-\mathbf{u}_{2}^{\lambda_{2}}, \mathbf{u}_{1}^{\boldsymbol{\eta}_{1}}-\mathbf{u}_{2}^{\eta_{2}}\right)_{\frac{1}{2}, \Gamma_{1}}+\frac{1}{2}\left(p_{2}^{\lambda_{2}}-p_{1}^{\boldsymbol{\lambda}_{1}}, p_{2}^{\eta_{2}}-p_{1}^{\boldsymbol{\eta}_{1}}\right)_{\frac{1}{2}, \Gamma_{2}}
$$

and the linear functional $\mathcal{L}: \boldsymbol{\Lambda} \rightarrow \mathbb{R}$

$$
\mathcal{L}(\boldsymbol{\eta})=-\frac{1}{2}\left(\mathbf{u}_{1}^{0, \mathbf{f}_{1}}-\mathbf{u}_{2}^{0, \mathbf{f}_{2}}, \mathbf{u}_{1}^{\boldsymbol{\eta}_{1}}-\mathbf{u}_{2}^{\eta_{2}}\right)_{\frac{1}{2}, \Gamma_{1}}-\frac{1}{2}\left(p_{2}^{0, \mathbf{f}_{2}}-p_{1}^{0, \mathbf{f}_{1}}, p_{2}^{\eta_{2}}-p_{1}^{\boldsymbol{\eta}_{1}}\right)_{\frac{1}{2}, \Gamma_{2}},
$$

so that (since $\mathbf{u}_{1}^{0, \mathbf{f}_{1}}=\mathbf{0}$ on $\Gamma_{1}$ and $p_{2}^{0, \mathbf{f}_{2}}=0$ on $\Gamma_{2}$ )

$$
J(\boldsymbol{\eta})=\pi(\boldsymbol{\eta}, \boldsymbol{\eta})-2 \mathcal{L}(\boldsymbol{\eta})+\frac{1}{2}\left\|\mathbf{u}_{2}^{0, \mathbf{f}_{2}}\right\|_{\frac{1}{2}, \Gamma_{1}}^{2}+\frac{1}{2}\left\|p_{1}^{0, \mathbf{f}_{1}}\right\|_{\frac{1}{2}, \Gamma_{2}}^{2} .
$$

It is immediate to show that $\pi$ and $\mathcal{L}$ are continuous in $\boldsymbol{\Lambda}$ w.r.t. the norm $\|\cdot\|$.

We prove now that the optimal control problem (3.13) has a unique solution.

THEOREM 3.5. Under the hypoteses of Lemma 3.4, problem (3.13), whose state variables satisfy (3.1), (3.2), has a unique solution $\boldsymbol{\lambda}=\underset{\boldsymbol{\eta} \in \mathbf{\Lambda}}{\operatorname{argmin}} J(\boldsymbol{\eta})$ that satisfies the Euler-Lagrange equation

$$
\begin{aligned}
\left\langle J^{\prime}(\boldsymbol{\lambda}), \boldsymbol{\eta}\right\rangle= & \left(\mathbf{u}_{1}^{\boldsymbol{\lambda}_{1}, \mathbf{f}_{1}}-\mathbf{u}_{2}^{\lambda_{2}, \mathbf{f}_{2}}, \mathbf{u}_{1}^{\boldsymbol{\eta}_{1}}-\mathbf{u}_{2}^{\eta_{2}}\right)_{\frac{1}{2}, \Gamma_{1}} \\
& +\left(p_{2}^{\lambda_{2}, \mathbf{f}_{2}}-p_{1}^{\boldsymbol{\lambda}_{1}, \mathbf{f}_{1}}, p_{2}^{\eta_{2}}-p_{1}^{\boldsymbol{\eta}_{1}}\right)_{\frac{1}{2}, \Gamma_{2}}=0 \quad \forall \boldsymbol{\eta} \in \boldsymbol{\Lambda} .
\end{aligned}
$$

Moreover, $\exists c>0$ constant such that $\|\boldsymbol{\lambda}\| \leq c\left(\left\|\mathbf{u}_{2}^{0, \mathbf{f}_{2}}\right\|_{\frac{1}{2}, \Gamma_{1}}+\left\|p_{1}^{0, \mathbf{f}_{1}}\right\|_{\frac{1}{2}, \Gamma_{2}}\right)$.

Proof. Thanks to Lemma 3.4, $\pi$ is coercive on $\boldsymbol{\Lambda}$ with respect to the norm $\|\cdot\|$ (as a matter of fact $\|\boldsymbol{\eta}\|^{2}=\pi(\boldsymbol{\eta}, \boldsymbol{\eta})$ ). Then, by applying classical results of the calculus of variations (see, e.g., [36, Thm I.1.1]), both existence and uniqueness of solution in $\boldsymbol{\Lambda}$ follow. The Euler-Lagrange equation (3.23) follows by noticing that $\left\langle J^{\prime}(\boldsymbol{\lambda}), \boldsymbol{\eta}\right\rangle=2 \pi(\boldsymbol{\lambda}, \boldsymbol{\eta})-2 \mathcal{L}(\boldsymbol{\eta})$, for any $\boldsymbol{\lambda}, \boldsymbol{\eta} \in \boldsymbol{\Lambda}$. Then $\boldsymbol{\lambda}$ is the unique solution of

$$
\pi(\boldsymbol{\lambda}, \boldsymbol{\eta})=\mathcal{L}(\boldsymbol{\eta}) \quad \forall \boldsymbol{\eta} \in \boldsymbol{\Lambda} .
$$

Finally, the last assertion follows from $\|\boldsymbol{\lambda}\|^{2}=\pi(\boldsymbol{\lambda}, \boldsymbol{\lambda})=\mathcal{L}(\boldsymbol{\lambda}) \leq c\left(\left\|\mathbf{u}_{2}^{0, \mathbf{f}_{2}}\right\|_{\frac{1}{2}, \Gamma_{1}}+\right.$ $\left.\left\|p_{1}^{0, \mathbf{f}_{1}}\right\|_{\frac{1}{2}, \Gamma_{2}}\right)\|\boldsymbol{\lambda}\|$. 
4. $h p$-FEM discretization. For $i=1,2$, let $\mathcal{T}_{i}$ be a partition of the computational domain $\Omega_{i} \subset \mathbb{R}^{d}$ in either simplices or quads (quadrilaterals when $d=2$ and hexahedra when $d=3$ ). The first ones are typical of classical FEM, the others of Spectral Elements Methods (SEM) with tensorial structure (see $[5,6]$ ); from now on we group them under $h p-\mathrm{FEM}$. We denote by $\hat{T}$ the reference element, that can be either the reference simplex with vertexes $\mathbf{0}$ and the points on the axis whose distance from the origin is 1 , or the $d$-dimensional cube $(-1,1)^{d}$.

We suppose that each element $T \in \mathcal{T}_{i}$ is obtained by a $C^{1}$ diffeomorphism $\mathbf{F}_{T}$ of the reference element $\hat{T}$ and we suppose that two adjacent elements of $\mathcal{T}_{i}$ share either a common vertex, or a complete edge, or else a complete face (when $d=3$ ). For each $T \in \mathcal{T}_{i}$ we denote by $h_{T}=\operatorname{diam}(T)=\max _{\mathbf{x}, \mathbf{y} \in T}|\mathbf{x}-\mathbf{y}|$ the diameter of element $T$ and we define $h_{i}=\max _{T \in \mathcal{T}_{i}} h_{T}$. Then, when simplicial partitions are considered, we require that the grid is regular in each $\Omega_{i}$ (see, e.g., [43]).

Given an integer $p \geq 1$, let us denote by $\mathbb{P}_{p}$ the space of polynomials whose global degree is less than or equal to $p$ in the variables $x_{1}, \ldots, x_{d}$ and by $\mathbb{Q}_{p}$ the space of polynomials that are of degree less than or equal to $p$ with respect to each variable $x_{1}, \ldots, x_{d}$. The space $\mathbb{P}_{p}$ is used for simplexes, $\mathbb{Q}_{p}$ for quads. We introduce the following finite dimensional spaces in $\bar{\Omega}_{i}$ :

$$
X_{i, h}^{p}=\left\{v \in C^{0}\left(\bar{\Omega}_{i}\right): v_{\mid T} \in \mathcal{Q}_{p}, \forall T \in \mathcal{T}_{i}\right\}
$$

where $\mathcal{Q}_{p}=\mathbb{P}_{p}$ in the simplicial case and $\mathcal{Q}_{p}=\mathbb{Q}_{p} \circ \mathbf{F}_{T}^{-1}$ for quads. Finally, let $\mathcal{M}_{i}$ be the set of the nodes $\mathbf{x}_{j}$ of the mesh $\mathcal{T}_{i}$.

In the Stokes subdomain we consider either inf-sup stable finite dimensional spaces or stabilized couples of spaces (see, e.g., $[2,19,21,25,42]$ ). In the Darcy subdomain we can either consider stabilized finite dimensional spaces (see, e.g., [38]) to approximate the velocity and the pressure, or adopt a Stokes-compatible formulation as that introduced in [11], or again inf-sup stable spaces. We assume that the polynomials used for both the Stokes velocity and the Darcy pressure are continuous (see, e.g., $[2,43])$ to guarantee that the traces of $\mathbf{u}_{1, h}$ and $p_{2, h}$ are well defined on $\Gamma_{1}$ and $\Gamma_{2}$, respectively. Then, for suitable polynomial degrees $p, r, t$ and $s$, the finite dimensional spaces for velocity and pressure are, respectively,

$$
\begin{aligned}
& \mathbf{V}_{1, h}=\mathbf{V}_{1} \cap\left[X_{1, h}^{p}\right]^{d}, \quad \mathbf{V}_{1, h}^{0}=\mathbf{V}_{1}^{0} \cap\left[X_{1, h}^{p}\right]^{d}, \quad Q_{1, h}=Q_{1} \cap X_{1, h}^{r}, \\
& \mathbf{V}_{2, h}=\mathbf{V}_{2} \cap\left[X_{2, h}^{t}\right]^{d}, \quad Q_{2, h}=Q_{2} \cap X_{2, h}^{s} \text {. }
\end{aligned}
$$

Finally, the spaces of discrete Dirichlet controls are defined as

$$
\begin{array}{r}
\boldsymbol{\Lambda}_{1, h}=\left\{\boldsymbol{\lambda}_{1, h} \in\left[C^{0}\left(\bar{\Gamma}_{1}\right)\right]^{d}: \exists \mathbf{v}_{1, h} \in \mathbf{V}_{1, h} \text { with } \boldsymbol{\lambda}_{1, h}=\left.\mathbf{v}_{1, h}\right|_{\Gamma_{1}}\right\} \subset \boldsymbol{\Lambda}_{1} \\
\Lambda_{2, h}=\left\{\lambda_{2, h} \in C^{0}\left(\bar{\Gamma}_{2}\right): \exists q_{2, h} \in Q_{2, h} \text { with } \lambda_{2, h}=\left.q_{2, h}\right|_{\Gamma_{2}}\right\} \subset \Lambda_{2} .
\end{array}
$$

We denote by $N_{\Omega_{1}}^{\mathbf{u}}, N_{\Omega_{1}}^{p}, N_{\Gamma_{1}}, N_{\Omega_{2}}^{\mathbf{u}}, N_{\Omega_{2}}^{p}$, and $N_{\Gamma_{2}}$ the cardinality of $\mathbf{V}_{1, h}^{0}, Q_{1, h}$, $\Lambda_{1, h}, \mathbf{V}_{2, h}, Q_{2, h}$, and $\Lambda_{2, h}$, respectively.

In each discrete functional space we consider the basis of the characteristic Lagrange polynomials associated with the nodes of $\mathcal{M}_{i}$ and we denote by $\varphi_{i, \ell}$ (for $i=1,2$ and $\ell=1, \ldots, N_{\Omega_{i}}^{\mathbf{u}}$ ) and $\psi_{i, \ell}$ (for $i=1,2$ and $\ell=1, \ldots, N_{\Omega_{i}}^{p}$ ) the basis functions of $\mathbf{V}_{1, h}^{0}, \mathbf{V}_{2, h}$ and $Q_{1, h}, Q_{2, h}$ respectively. The basis functions in $\boldsymbol{\Lambda}_{1, h}$ are denoted by $\boldsymbol{\eta}_{1, \ell}$ (for $\ell=1, \ldots, N_{\Gamma_{1}}$ ) and they are defined by restriction to $\Gamma_{1}$ of the basis functions of $\mathbf{V}_{1, h}$ that are not identically null on $\Gamma_{1}$. Similarly we define the basis function $\eta_{2, \ell}$ (for $\ell=1, \ldots, N_{\Gamma_{2}}$ ) of $\Lambda_{2, h}$, starting from the basis in $Q_{2, h}$. 
Because of the difficulty to compute integrals exactly for large $p$, typically when quad partitions are employed, Legendre-Gauss-Lobatto quadrature formulas are used to approximate both the bilinear forms $\mathcal{A}_{i}$ and the $L^{2}$-inner products in $\Omega_{i}$ (as well as on the interfaces). This leads to the so-called Galerkin approach with Numerical Integration (G-NI) [5, 4] and to the Spectral Element Method with Numerical Integration (SEM-NI).

We define the discrete counterpart of the extension operator: $E_{1, h}: \boldsymbol{\Lambda}_{1, h} \rightarrow \mathbf{V}_{1, h}$ s.t. $E_{1, h} \boldsymbol{\lambda}_{1, h}=\boldsymbol{\lambda}_{1, h}$ on $\Gamma_{1}$ and $\left(E_{1, h} \boldsymbol{\lambda}_{1, h}\right)\left(\mathbf{x}_{j}\right)=\mathbf{0}$ for any $\mathbf{x}_{j} \in \mathcal{M}_{1} \cap\left(\bar{\Omega}_{1} \backslash\left(\Gamma_{1} \cup \partial \Omega_{1}^{N}\right)\right)$. The discrete counterpart of (3.1)-(3.2) reads: given $\mathbf{f}_{1} \in\left[L^{2}\left(\Omega_{1}\right)\right]^{d}, \mathbf{f}_{2} \in H\left(\operatorname{div}, \Omega_{2}\right)$, $\boldsymbol{\lambda}_{1, h} \in \boldsymbol{\Lambda}_{1, h}, \lambda_{2, h} \in \Lambda_{2, h}$ we look for $\left(\mathbf{u}_{1,0, h}, p_{1, h}\right) \in \mathbf{V}_{1, h}^{0} \times Q_{1, h}$ and $\left(\mathbf{u}_{2, h}, p_{2, h}\right) \in$ $\mathbf{V}_{2, h} \times Q_{2, h}$ such that

$$
\begin{aligned}
& \mathcal{A}_{1, h}\left(\mathbf{u}_{1,0, h}, p_{1, h} ; \mathbf{v}_{1, h}, q_{1, h}\right)=-\mathcal{B}_{1, h}\left(E_{1, h} \boldsymbol{\lambda}_{1, h} ; \mathbf{v}_{1, h}, q_{1, h}\right)+\mathcal{F}_{1, h}\left(\mathbf{v}_{1, h}, q_{1, h}\right) \\
& \\
& \forall\left(\mathbf{v}_{1, h}, q_{1, h}\right) \in \mathbf{V}_{1, h}^{0} \times Q_{1, h} \\
& \mathbf{u}_{1, h}=\mathbf{u}_{1,0, h}+E_{1, h} \boldsymbol{\lambda}_{1, h} \\
& \mathcal{A}_{2, h}\left(\mathbf{u}_{2, h}, p_{2, h} ; \mathbf{v}_{2, h}, q_{2, h}\right)=-\mathcal{B}_{2, h}\left(\lambda_{2, h} ; \mathbf{v}_{2, h}\right)+ \mathcal{F}_{2, h}\left(\mathbf{v}_{2, h}, q_{2, h}\right) \\
& \forall\left(\mathbf{v}_{2, h}, q_{2, h}\right) \in \mathbf{V}_{2, h} \times Q_{2, h}
\end{aligned}
$$

where, for $\gamma_{1} \in\{-1,0,1\}$, and $\tau_{k}=\tau_{k}(\mathbf{x})$ a stabilization parameter depending on both mesh size $h$ and local polynomial degree (see, e.g. [19, 21]), we set:

$$
\begin{aligned}
& \mathcal{A}_{1, h}\left(\mathbf{w}_{1, h}, s_{1, h} ; \mathbf{v}_{1, h}, q_{1, h}\right)=a_{1}\left(\mathbf{w}_{1, h}, \mathbf{v}_{1, h}\right)+b_{1}\left(\mathbf{v}_{1, h}, s_{1, h}\right)-b_{1}\left(\mathbf{w}_{1, h}, q_{1, h}\right) \\
& \quad+\sum_{T_{k} \in \mathcal{T}_{1}}\left(-\nu \Delta \mathbf{w}_{1, h}+\nabla s_{1, h}, \tau_{k}\left(\gamma_{1} \nu \Delta \mathbf{v}_{1, h}+\nabla q_{1, h}\right)\right)_{L^{2}\left(T_{k}\right)} \\
& \mathcal{B}_{1, h}\left(\mathbf{w}_{1, h} ; \mathbf{v}_{1, h}, q_{1, h}\right)=\mathcal{A}_{1, h}\left(\mathbf{w}_{1, h}, 0 ; \mathbf{v}_{1, h}, q_{1, h}\right) \\
& \mathcal{F}_{1, h}\left(\mathbf{v}_{1, h}, q_{1, h}\right)=\mathcal{F}_{1}\left(\mathbf{v}_{1, h}\right)+\sum_{T_{k} \in \mathcal{T}_{1}}\left(\mathbf{f}_{1}, \tau_{k}\left(\gamma_{1} \nu \Delta \mathbf{v}_{1, h}+\nabla q_{1, h}\right)\right)_{L^{2}\left(T_{k}\right)} .
\end{aligned}
$$

Moreover,

$$
\begin{aligned}
& \mathcal{A}_{2, h}\left(\mathbf{w}_{2, h}, s_{2, h} ; \mathbf{v}_{2, h}, q_{2, h}\right)=a_{2}\left(\mathbf{w}_{2, h}, \mathbf{v}_{2, h}\right)+b_{2}\left(\mathbf{v}_{2, h}, s_{2, h}\right)-b_{2}\left(\mathbf{w}_{2, h}, q_{2, h}\right) \\
& \quad+\gamma_{2,1}\left(\boldsymbol{\alpha}^{-1} \mathbf{w}_{2, h}+\nabla s_{2, h}, \boldsymbol{\alpha} / 2\left(-\boldsymbol{\alpha}^{-1} \mathbf{v}_{2, h}+\nabla q_{2, h}\right)\right)_{L^{2}\left(\Omega_{2}\right)} \\
& \quad+\gamma_{2,2}\left(\nabla \cdot \mathbf{w}_{2, h},\left\|\boldsymbol{\alpha}^{-1}\right\| \nabla \cdot \mathbf{v}_{2, h}\right)_{L^{2}\left(\Omega_{2}\right)}+\gamma_{2,3}\left(\boldsymbol{\alpha} \nabla \times\left(\boldsymbol{\alpha}^{-1} \mathbf{w}_{2, h}\right), \nabla \times\left(\boldsymbol{\alpha}^{-1} \mathbf{v}_{2, h}\right)\right)_{L^{2}\left(\Omega_{2}\right)}, \\
& \mathcal{B}_{2, h}\left(s_{2, h} ; \mathbf{v}_{2, h}\right)=\left\langle\mathbf{v}_{2, h} \cdot \mathbf{n}_{2}, s_{2, h}\right\rangle_{\Gamma_{2}} \\
& \mathcal{F}_{2, h}\left(\mathbf{v}_{2, h}, q_{2, h}\right)=\mathcal{F}_{2}\left(\mathbf{v}_{2, h}\right)+\gamma_{2,1}\left(\mathbf{f}_{2}, \boldsymbol{\alpha} / 2\left(-\boldsymbol{\alpha}^{-1} \mathbf{v}_{2, h}+\nabla q_{2, h}\right)\right)_{L^{2}\left(\Omega_{2}\right)} \\
& \quad+\gamma_{2,3}\left(\boldsymbol{\alpha} \nabla \times\left(\boldsymbol{\alpha}^{-1} \mathbf{f}_{2}\right), \nabla \times\left(\boldsymbol{\alpha}^{-1} \mathbf{v}_{2, h}\right)\right)_{L^{2}\left(\Omega_{2}\right)} .
\end{aligned}
$$

For $\gamma_{2,1}=1, \gamma_{2,2}=h^{2} / 2, \gamma_{2,3}=0$ we recover the stabilization method [38], while if $\gamma_{2,1}=0$ and $\gamma_{2,2}$ and $\gamma_{2,3}$ are suitably chosen parameters we obtain the formulation proposed in [11]. If $\tau_{k}=0$ in (4.5) we recover the classical weak form of the Stokes problem, that is well-posed once the discrete spaces satisfy the inf-sup condition [2]. We need however to mention that other finite dimensional discretizations could be used as well. As a matter of fact, the emphasis of this paper is on the ICDD modeling and not necessarily on the specific way the two subproblems (Stokes' and Darcy's) are approximated.

As for the continuous case, the solution of (4.4) depends on both the control $\boldsymbol{\lambda}_{h}$ and the given function $\mathbf{f}$, thus actually we have $\mathbf{u}_{1, h}=\mathbf{u}_{1, h}^{\boldsymbol{\lambda}_{1, h}, \mathbf{f}_{1}}, p_{1, h}=p_{1, h}^{\boldsymbol{\lambda}_{1, h}, \mathbf{f}_{1}}$, 
$\mathbf{u}_{2, h}=\mathbf{u}_{2, h}^{\lambda_{2, h}, \mathbf{f}_{2}}$, and $p_{2, h}=p_{2, h}^{\lambda_{2, h}, \mathbf{f}_{2}}$, and $\mathbf{u}_{1, h}=\mathbf{u}_{1, h}^{\boldsymbol{\lambda}_{1, h}}, p_{1, h}=p_{1, h}^{\boldsymbol{\lambda}_{1, h}}, \mathbf{u}_{2, h}=\mathbf{u}_{2, h}^{\lambda_{2, h}}$, and $p_{2, h}=p_{2, h}^{\lambda_{2, h}}$, when $\mathbf{f}_{1}=\mathbf{0}$ and $\mathbf{f}_{2}=\mathbf{0}$.

Let us define $\boldsymbol{\Lambda}_{h}=\boldsymbol{\Lambda}_{1, h} \times \Lambda_{2, h}$ and for any $\boldsymbol{\eta}_{h} \in \boldsymbol{\Lambda}_{h}$ let $\boldsymbol{\eta}_{h}=\left(\boldsymbol{\eta}_{1, h}, \eta_{2, h}\right)$.

The discrete counterpart of the minimization problem (2.2) reads: look for

$$
\begin{aligned}
\boldsymbol{\lambda}_{h}=\underset{\boldsymbol{\eta}_{h} \in \boldsymbol{\Lambda}_{h}}{\operatorname{argmin}}\left[J_{h}\left(\boldsymbol{\eta}_{h}\right)=\right. & \frac{1}{2}\left\|\mathbf{u}_{1, h}^{\boldsymbol{\eta}_{1, h}, \mathbf{f}_{1}}-\mathcal{P}_{1}\left(\mathbf{u}_{2, h}^{\eta_{2, h}, \mathbf{f}_{2}}\right)\right\|_{\frac{1}{2}, \Gamma_{1}}^{2} \\
& \left.+\frac{1}{2}\left\|p_{2, h}^{\eta_{2, h}, \mathbf{f}_{2}}-\mathcal{P}_{2}\left(p_{1, h}^{\boldsymbol{\eta}_{1, h}, \mathbf{f}_{1}}\right)\right\|_{\frac{1}{2}, \Gamma_{2}}^{2}\right],
\end{aligned}
$$

where $\left(\mathbf{u}_{1, h}^{\boldsymbol{\eta}_{1, h}, \mathbf{f}_{1}}, p_{1, h}^{\boldsymbol{\eta}_{1, h}, \mathbf{f}_{1}}\right)$ and $\left(\mathbf{u}_{2, h}^{\eta_{2, h}, \mathbf{f}_{2}}, p_{2, h}^{\eta_{2, h}, \mathbf{f}_{2}}\right)$ are the solutions of (4.4) with $\boldsymbol{\eta}_{h}$ instead of $\boldsymbol{\lambda}_{h}$, while $\mathcal{P}_{1}$ is a suitable projection/interpolation operator from $\mathbf{V}_{2, h}$ to $\boldsymbol{\Lambda}_{1, h}$ and $\mathcal{P}_{2}$ is a suitable projection/interpolation operator from $Q_{1, h}$ to $\Lambda_{2, h}$. Should the two discretizations coincide on the overlap, then $\mathcal{P}_{1}$ and $\mathcal{P}_{2}$ would be the identity operator.

In order to prove that the minimization problem (4.6) admits a unique solution we proceed as in the previous section.

For any $\boldsymbol{\lambda}_{h}=\left(\boldsymbol{\lambda}_{1, h}, \lambda_{2, h}\right) \in \boldsymbol{\Lambda}_{h}$, with $\left(\mathbf{u}_{1, h}^{\boldsymbol{\lambda}_{1, h}}, p_{1}^{\boldsymbol{\lambda}_{1, h}}\right)$ and $\left(\mathbf{u}_{2, h}^{\lambda_{2, h}}, p_{2, h}^{\lambda_{2, h}}\right)$ solutions of (4.4) with $\mathbf{f}_{i}=\mathbf{0}$ for $i=1,2$, we define the seminorm

$$
\left\|\boldsymbol{\lambda}_{h}\right\|_{h}=\left(\left\|\mathbf{u}_{1, h}^{\lambda_{1, h}}-\mathcal{P}_{1}\left(\mathbf{u}_{2, h}^{\lambda_{2, h}}\right)\right\|_{\frac{1}{2}, \Gamma_{1}}^{2}+\left\|p_{2, h}^{\lambda_{2, h}}-\mathcal{P}_{2}\left(p_{1, h}^{\boldsymbol{\lambda}_{1, h}}\right)\right\|_{\frac{1}{2}, \Gamma_{2}}^{2}\right)^{1 / 2}
$$

LEMMA 4.1. Let (3.12) and Assumptions 2.1 be satisfied. If the discrete spaces $\mathbf{V}_{i, h}$ and $Q_{i, h}$, for $i=1,2$, are suitably chosen to guarantee the well-posedness of the discrete problems (4.4), then $\|\cdot\|_{h}$ is indeed a norm on $\boldsymbol{\Lambda}_{h}$.

Proof. We only have to prove that $\left\|\boldsymbol{\lambda}_{h}\right\|_{h}=0$ implies $\boldsymbol{\lambda}_{h}=\mathbf{0}$.

We observe that $\left\|\boldsymbol{\lambda}_{h}\right\|_{h}=0$ implies that the solution of (4.4) with $\mathbf{f}_{1}=\mathbf{0}$ and $\mathbf{f}_{2}=\mathbf{0}$ satisfies $\left(\boldsymbol{\lambda}_{1, h}=\right) \mathbf{u}_{1, h}^{\boldsymbol{\lambda}_{1, h}}=\mathcal{P}_{1}\left(\mathbf{u}_{2, h}^{\lambda_{2, h}}\right)$ on $\Gamma_{1}$ and $\left(\lambda_{2, h}=\right) p_{2, h}^{\lambda_{2, h}}=\mathcal{P}_{2}\left(p_{1, h}^{\boldsymbol{\lambda}_{1, h}}\right)$ on $\Gamma_{2}$, i.e. it is the solution of

$$
\begin{array}{lr}
\mathcal{A}_{1, h}\left(\mathbf{u}_{1,0, h}^{\boldsymbol{\lambda}_{1, h}}, p_{1, h}^{\boldsymbol{\lambda}_{1, h}} ; \mathbf{v}_{1, h}, q_{1, h}\right)=-\mathcal{B}_{1, h}\left(E_{1, h}\left(\boldsymbol{\lambda}_{1, h}\right) ; \mathbf{v}_{1, h}, q_{1, h}\right) & \forall\left(\mathbf{v}_{1, h}, q_{1, h}\right) \in \mathbf{V}_{1, h}^{0} \times Q_{1, h} \\
\mathbf{u}_{1, h}^{\boldsymbol{\lambda}_{1, h}}=\mathbf{u}_{1,0, h}^{\boldsymbol{\lambda}_{1, h}}+E_{1, h}\left(\boldsymbol{\lambda}_{1, h}\right) & \\
\boldsymbol{\lambda}_{1, h}=\mathcal{P}_{1}\left(\mathbf{u}_{2, h}^{\lambda_{2, h}}\right) & \\
\mathcal{A}_{2, h}\left(\mathbf{u}_{2, h}^{\lambda_{2, h}}, p_{2, h}^{\lambda_{2, h}} ; \mathbf{v}_{2, h}, q_{2, h}\right)=-\mathcal{B}_{2, h}\left(\lambda_{2, h} ; \mathbf{v}_{2, h}\right) & \\
\lambda_{2, h}=\mathcal{P}_{2}\left(p_{1, h}^{\boldsymbol{\lambda}_{1, h}}\right) . &
\end{array}
$$

In matrix form (4.8) reads

$$
\underbrace{\left[\begin{array}{cc|cc}
\mathrm{A}_{1} & \mathrm{~B}_{1} & 0 & 0 \\
0 & I & -\mathrm{P}_{1} & 0 \\
\hline 0 & 0 & \mathrm{~A}_{2} & \mathrm{~B}_{2} \\
-\mathrm{P}_{2} & 0 & 0 & I
\end{array}\right]}_{G}\left[\begin{array}{c}
\underline{\mathbf{U}}_{1} \\
\underline{\boldsymbol{\lambda}}_{1} \\
\hline \underline{\mathbf{U}}_{2} \\
\underline{\boldsymbol{\lambda}}_{2}
\end{array}\right]=\left[\begin{array}{l}
\mathbf{0} \\
\mathbf{0} \\
\hline \mathbf{0} \\
\mathbf{0}
\end{array}\right],
$$

where:

- $\underline{\mathbf{U}}_{i} \in \mathbb{R}^{N_{\Omega_{i}}}$ contains the d.o.f. of both velocity and pressure in $\Omega_{i}$;

- $\underline{\boldsymbol{\lambda}}_{i} \in \mathbb{R}^{N_{\Gamma_{i}}}$ contains the d.o.f. of the control function on $\Gamma_{i}$;

- $\mathrm{A}_{i}$ is the matrix associated with the bilinear form $\mathcal{A}_{i, h}$;

- $\mathrm{B}_{i}$ is the matrix associated with the bilinear form $\mathcal{B}_{i, h}$; 
- $\mathrm{P}_{i}$ are the matrices that perform the projection/interpolation of $\left(\mathbf{u}_{j}, p_{j}\right)$ (only for the part involving either the velocity d.o.f. in the Darcy domain or the pressure ones in the Stokes domain) to $\Gamma_{i}$.

Clearly $\boldsymbol{\lambda}_{h}=\mathbf{0}$ is a solution of (4.8). We have to prove that the unique solution of (4.8) is the null one. To this aim we prove that the rows of $G$ are linearly independent. We split the matrix $G$ in $2 \times 2$ blocks $G_{i j}(i, j=1,2)$ of size $N_{i} \times N_{j}$, as in (4.9), and we indentify four blocks of rows: $G 1=\left[\mathrm{A}_{1}, \mathrm{~B}_{1}, 0,0\right], G 2=\left[0, I,-\mathrm{P}_{1}, 0\right]$, $G 3=\left[0,0, \mathrm{~A}_{2}, \mathrm{~B}_{2}\right]$, and $G 4=\left[-\mathrm{P}_{2}, 0,0, I\right]$.

Since the Stokes and Darcy subproblems are well-posed by hypothesis, blocks $G_{i i}$ are non-singular, and all the row-blocks $G 1, \ldots, G 4$ are full rank. Moreover, in view of the definition of $G$, it is easy to verify that: the rows of $G 1$ and $G 2$ are linearly independent, as well as those of $G 3$ and $G 4$, those of $G 1$ and $G 3$, and those of $G 2$ and $G 4$. It remains to prove that the rows of $G 2$ and $G 3$ are linearly independent, as well as those of $G 1$ and $G 4$. Let us consider an arbitrary row $\mathbf{v}_{i}$ of $G 2$ and an arbitrary row $\mathbf{w}_{j}$ of $G 3$. We can write them as

$$
\begin{aligned}
& \mathbf{v}_{i}=\left[\begin{array}{lll}
0 \ldots 0, & 0 \ldots 1 \ldots 0, \quad-\mathrm{P}_{1}, \quad 0 \ldots 0
\end{array}\right],
\end{aligned}
$$

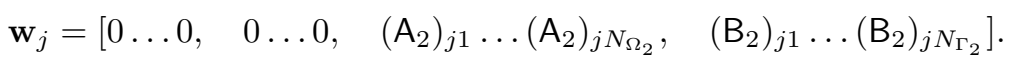

Let $\alpha, \beta \in \mathbb{R}$, then $\alpha \mathbf{v}_{i}+\beta \mathbf{w}_{j}=\mathbf{0}$ immediately implies $\alpha=0$ and thus $\beta=0$. In view of the arbitrary choice of $\mathbf{v}_{i}$ in $G 2$ and $\mathbf{w}_{j}$ in $G 3$, it follows that all the rows of $G 2$ and $G 3$ are linearly independent. By using the same arguments, it holds that the rows of $G 1$ and $G 4$ are linearly independent, so $G$ is full rank.

The following theorem is the counterpart of Theorem 3.5 and it ensures the existence and uniqueness of the solution of the minimization problem (4.6).

TheOREM 4.2. Let (3.12) and Assumptions 2.1 be satisfied. If moreover the discrete spaces $\mathbf{V}_{i, h}$ and $Q_{i, h}$, for $i=1,2$, are suitably chosen to guarantee the wellposedness of the discrete problems (4.4), then the discrete minimization problem (4.6) has a unique solution $\boldsymbol{\lambda}_{h} \in \boldsymbol{\Lambda}_{h}$ satisfying the Euler-Lagrange equation

$$
\begin{aligned}
\left\langle J_{h}^{\prime}\left(\boldsymbol{\lambda}_{h}\right), \boldsymbol{\eta}_{h}\right\rangle= & \left(\mathbf{u}_{1, h}^{\boldsymbol{\lambda}_{1, h}, \mathbf{f}_{1}}-\mathcal{P}_{1}\left(\mathbf{u}_{2, h}^{\lambda_{2, h}, \mathbf{f}_{2}}\right), \mathbf{u}_{1, h}^{\boldsymbol{\eta}_{1, h}}-\mathcal{P}_{1}\left(\mathbf{u}_{2, h}^{\eta_{2, h}}\right)\right)_{\frac{1}{2}, \Gamma_{1}} \\
& +\left(p_{2, h}^{\lambda_{2, h}, \mathbf{f}_{2}}-\mathcal{P}_{2}\left(p_{1, h}^{\boldsymbol{\lambda}_{1, h}, \mathbf{f}_{1}}\right), p_{2, h}^{\eta_{2, h}}-\mathcal{P}_{2}\left(p_{1, h}^{\boldsymbol{\eta}_{1, h}}\right)\right)_{\frac{1}{2}, \Gamma_{2}}=0
\end{aligned}
$$

where $\left(\mathbf{u}_{1, h}^{\boldsymbol{\lambda}_{1, h}, \mathbf{f}_{1}}, p_{1, h}^{\boldsymbol{\lambda}_{1, h}, \mathbf{f}_{1}}\right)$ and $\left(\mathbf{u}_{2, h}^{\lambda_{2, h}, \mathbf{f}_{2}}, p_{2, h}^{\lambda_{2, h}, \mathbf{f}_{2}}\right)$ are the solutions of (4.4). Moreover, $\exists c \in \mathbb{R}^{+}$independent of the discretization s.t. $\left\|\boldsymbol{\lambda}_{h}\right\|_{h} \leq c\left(\left\|\mathbf{u}_{2, h}^{0, \mathbf{f}_{2}}\right\|_{\frac{1}{2}, \Gamma_{1}}+\left\|p_{1, h}^{0, \mathbf{f}_{1}}\right\|_{\frac{1}{2}, \Gamma_{2}}\right)$.

Proof. The proof is as in Theorem 3.5 with:

$$
\begin{aligned}
\pi_{h}\left(\boldsymbol{\lambda}_{h}, \boldsymbol{\eta}_{h}\right)= & \frac{1}{2}\left(\mathbf{u}_{1, h}^{\boldsymbol{\lambda}_{1, h}}-\mathcal{P}_{1}\left(\mathbf{u}_{2, h}^{\lambda_{2, h}}\right), \mathbf{u}_{1, h}^{\boldsymbol{\eta}_{1, h}}-\mathcal{P}_{1}\left(\mathbf{u}_{2, h}^{\eta_{2, h}}\right)\right)_{\frac{1}{2}, \Gamma_{1}} \\
& +\frac{1}{2}\left(p_{2, h}^{\lambda_{2, h}}-\mathcal{P}_{2}\left(p_{1, h}^{\boldsymbol{\lambda}_{1, h}}\right), p_{2, h}^{\eta_{2, h}}-\mathcal{P}_{2}\left(p_{1, h}^{\boldsymbol{\eta}_{1, h}}\right)\right)_{\frac{1}{2}, \Gamma_{2}}
\end{aligned}
$$

(a bilinear, continuous and coercive form on $\boldsymbol{\Lambda}_{h}$ w.r.t. the norm $\|\cdot\|_{h}$ ), and

$$
\begin{aligned}
\mathcal{L}_{h}\left(\boldsymbol{\eta}_{h}\right)=- & \frac{1}{2}\left(\mathbf{u}_{1, h}^{0, \mathbf{f}_{1}}-\mathcal{P}_{1}\left(\mathbf{u}_{2, h}^{0, \mathbf{f}_{2}}\right), \mathbf{u}_{1, h}^{\boldsymbol{\eta}_{1, h}}-\mathcal{P}_{1}\left(\mathbf{u}_{2, h}^{\eta_{2, h}}\right)_{\frac{1}{2}, \Gamma_{1}}\right. \\
& -\frac{1}{2}\left(p_{2, h}^{0, \mathbf{f}_{2}}-\mathcal{P}_{2}\left(p_{1, h}^{0, \mathbf{f}_{1}}\right), p_{2, h}^{\eta_{2}}-\mathcal{P}_{2}\left(p_{1, h}^{\boldsymbol{\eta}_{1, h}}\right)\right)_{\frac{1}{2}, \Gamma_{2}}
\end{aligned}
$$

(a linear and continuous functional on $\boldsymbol{\Lambda}_{h}$ w.r.t. the norm $\|\cdot\|_{h}$ ). Since $\mathbf{u}_{1, h}^{0, \mathbf{f}_{1}}=\mathbf{0}$ on $\Gamma_{1}$ and $p_{2, h}^{0, \mathbf{f}_{2}}=0$ on $\Gamma_{2}, J_{h}\left(\boldsymbol{\eta}_{h}\right)=\pi_{h}\left(\boldsymbol{\eta}_{h}, \boldsymbol{\eta}_{h}\right)-2 \mathcal{L}_{h}\left(\boldsymbol{\eta}_{h}\right)+\frac{1}{2}\left\|\mathbf{u}_{2, h}^{0, \mathbf{f}_{2}}\right\|_{\frac{1}{2}, \Gamma_{1}}^{2}+\frac{1}{2}\left\|p_{1, h}^{0, \mathbf{f}_{1}}\right\|_{\frac{1}{2}, \Gamma_{2}}^{2}$. 
The Euler-Lagrange equation (4.10) and the stability estimate follow by noticing that $\left\langle J_{h}^{\prime}\left(\boldsymbol{\lambda}_{h}\right), \boldsymbol{\eta}_{h}\right\rangle=2 \pi_{h}\left(\boldsymbol{\lambda}_{h}, \boldsymbol{\eta}_{h}\right)-2 \mathcal{L}_{h}\left(\boldsymbol{\eta}_{h}\right)$, for any $\boldsymbol{\lambda}_{h}, \boldsymbol{\eta}_{h} \in \boldsymbol{\Lambda}_{h}$, that is $\boldsymbol{\lambda}_{h}$ is the unique solution of

$$
\pi_{h}\left(\boldsymbol{\lambda}_{h}, \boldsymbol{\eta}_{h}\right)=\mathcal{L}_{h}\left(\boldsymbol{\eta}_{h}\right) \quad \forall \boldsymbol{\eta}_{h} \in \boldsymbol{\Lambda}_{h}
$$

$\square$

The Euler-Lagrange equation (4.10) can be reformulated in an equivalent way by a set of interface conditions that will be fundamental in designing the optimality system associated with the minimization problem (4.6). We prove such equivalence in the following theorem.

THEOREM 4.3. Let the assumptions of Theorem 4.2 be satisfied. $\boldsymbol{\lambda}_{h}$ is the solution of (4.10) if and only if

$$
\begin{array}{lr}
\left(\mathbf{u}_{1, h}^{\boldsymbol{\lambda}_{1, h}, \mathbf{f}_{1}}-\mathcal{P}_{1}\left(\mathbf{u}_{2, h}^{\lambda_{2, h}, \mathbf{f}_{2}}\right), \boldsymbol{\eta}_{1, h}\right)_{\frac{1}{2}, \Gamma_{1}}=0 & \forall \boldsymbol{\eta}_{1, h} \in \boldsymbol{\Lambda}_{1, h}, \\
\left(p_{2, h}^{\lambda_{2, h}, \mathbf{f}_{2}}-\mathcal{P}_{2}\left(p_{1, h}^{\boldsymbol{\lambda}_{1, h}, \mathbf{f}_{1}}\right), \eta_{2, h}\right)_{\frac{1}{2}, \Gamma_{2}}=0 & \forall \eta_{2, h} \in \boldsymbol{\Lambda}_{2, h} .
\end{array}
$$

Correspondingly, $J_{h}\left(\boldsymbol{\lambda}_{h}\right)=0$.

Proof. Let us define the linear map $\psi_{h}: \boldsymbol{\Lambda}_{h} \rightarrow \boldsymbol{\Lambda}_{h}$ such that

$$
\boldsymbol{\eta}_{h}=\left[\begin{array}{c}
\boldsymbol{\eta}_{1, h} \\
\eta_{2, h}
\end{array}\right] \mapsto\left[\begin{array}{c}
\left(\mathbf{u}_{1, h}^{\boldsymbol{\eta}_{1, h}}, p_{1, h}^{\boldsymbol{\eta}_{1, h}}\right) \\
\left(\mathbf{u}_{2, h}^{\eta_{2, h}}, p_{2, h}^{\eta_{2, h}}\right)
\end{array}\right] \mapsto\left[\begin{array}{c}
\mathcal{P}_{1}\left(\mathbf{u}_{2, h}^{\eta_{2, h}}\right) \\
\mathcal{P}_{2}\left(p_{1, h}^{\boldsymbol{\eta}_{1, h}}\right)
\end{array}\right]=\psi_{h}\left(\boldsymbol{\eta}_{h}\right) .
$$

$\boldsymbol{\lambda}_{h}=\mathbf{0}$ is clearly the only fixed point of $\psi_{h}$ thanks to Lemma 4.1 , thus the map $\tilde{\psi}_{h}: \boldsymbol{\Lambda}_{h} \rightarrow \boldsymbol{\Lambda}_{h}:$

$$
\tilde{\psi}_{h}\left(\boldsymbol{\eta}_{h}\right)=\boldsymbol{\eta}_{h}-\psi_{h}\left(\boldsymbol{\eta}_{h}\right)=\left[\begin{array}{c}
\boldsymbol{\eta}_{1, h}-\mathcal{P}_{1}\left(\mathbf{u}_{2, h}^{\eta_{2, h}}\right) \\
\eta_{2, h}-\mathcal{P}_{2}\left(p_{1, h}^{\boldsymbol{\eta}_{1, h}}\right)
\end{array}\right]=\left[\begin{array}{c}
\mathbf{u}_{1, h}^{\boldsymbol{\eta}_{1, h}, \mathbf{f}_{1}}-\mathcal{P}_{1}\left(\mathbf{u}_{2, h}^{\eta_{2, h}}\right) \\
p_{2, h}^{\eta_{2, h}, \mathbf{f}_{2}}-\mathcal{P}_{2}\left(p_{1, h}^{\boldsymbol{\eta}_{1, h}}\right)
\end{array}\right]
$$

is injective and therefore surjective. This implies the equivalence between (4.10) and (4.12). Moreover, the interface conditions (4.12) hold also in strong form, i.e.,

$$
\mathbf{u}_{1, h}^{\boldsymbol{\lambda}_{1, h}, \mathbf{f}_{1}}{ }_{\mid \Gamma_{1}}=\mathcal{P}_{1}\left(\mathbf{u}_{2, h}^{\lambda_{2, h}, \mathbf{f}_{2}}\right), \quad p_{2, h}^{\boldsymbol{\lambda}_{2, h}, \mathbf{f}_{2}}{ }_{\mid \Gamma_{2}}=\mathcal{P}_{2}\left(p_{1, h}^{\lambda_{1, h}, \mathbf{f}_{1}}\right),
$$

hence $J_{h}\left(\boldsymbol{\lambda}_{h}\right)=0$.

4.1. Convergence analysis. The following theorem states the convergence of the discrete solution of (4.6) to the solution of (2.2).

THEOREM 4.4. Let (3.12) and Assumptions 2.1 be satisfied, and $\boldsymbol{\lambda}, \boldsymbol{\lambda}_{h}$ be the solutions of (2.2) and (4.6), respectively. There exists $\widetilde{C}>0$ depending both on the geometry and on the model parameters s.t.

$$
\begin{aligned}
\left\|\boldsymbol{\lambda}-\boldsymbol{\lambda}_{h}\right\| \leq & C\left(\inf _{\boldsymbol{\eta}_{1, h} \in \boldsymbol{\Lambda}_{1, h}}\left\|\boldsymbol{\lambda}_{1}-\boldsymbol{\eta}_{1, h}\right\|_{\frac{1}{2}, \Gamma_{1}}+\inf _{\eta_{2, h} \in \Lambda_{2, h}}\left\|\lambda_{2}-\eta_{2, h}\right\|_{\frac{1}{2}, \Gamma_{2}}\right) \\
& +\sup _{\boldsymbol{\eta}_{1, h} \in \boldsymbol{\Lambda}_{1, h}}\left\|p_{1}^{\boldsymbol{\eta}_{1, h}, \mathbf{f}_{1}}-\mathcal{P}_{2}\left(p_{1, h}^{\boldsymbol{\eta}_{1, h}, \mathbf{f}_{1}}\right)\right\|_{\frac{1}{2}, \Gamma_{2}}+\sup _{\eta_{2, h} \in \Lambda_{2}, h}\left\|\mathbf{u}_{2}^{\eta_{2, h}, \mathbf{f}_{2}}-\mathcal{P}_{1}\left(\mathbf{u}_{2, h}^{\eta_{2, h}, \mathbf{f}_{2}}\right)\right\|_{\frac{1}{2}, \Gamma_{1}} .
\end{aligned}
$$

Proof. Let $\boldsymbol{\sigma}_{h}=\boldsymbol{\lambda}_{h}-\boldsymbol{\eta}_{h}$, for any arbitrary $\boldsymbol{\eta}_{h} \in \boldsymbol{\Lambda}_{h}$. Recalling the definition of $\pi$, that $\boldsymbol{\lambda}$ and $\boldsymbol{\lambda}_{h}$ are the solutions of (3.24) and (4.11), respectively, that $\boldsymbol{\Lambda}_{h} \subset \boldsymbol{\Lambda}$, the 
strong interface conditions (4.14) and noticing that $\mathbf{u}_{1}^{\boldsymbol{\lambda}_{1, h}, \mathbf{f}_{1}}{ }_{\mid \Gamma_{1}}=\mathbf{u}_{1, h}^{\boldsymbol{\lambda}_{1, h}, \mathbf{f}_{1}}{ }_{\mid \Gamma_{1}}=\boldsymbol{\lambda}_{1, h}$ and $p_{2}^{\lambda_{2, h}, \mathbf{f}_{2}}{ }_{\mid \Gamma_{2}}=p_{2, h}^{\lambda_{2, h}, \mathbf{f}_{2}}{ }_{\mid \Gamma_{2}}=\lambda_{2, h}$, it holds

$$
\begin{aligned}
& \left\|\boldsymbol{\sigma}_{h}\right\|^{2}=\pi\left(\boldsymbol{\sigma}_{h}, \boldsymbol{\sigma}_{h}\right)=\pi\left(\boldsymbol{\lambda}_{h}, \boldsymbol{\sigma}_{h}\right)-\pi\left(\boldsymbol{\eta}_{h}, \boldsymbol{\sigma}_{h}\right)+\pi\left(\boldsymbol{\lambda}, \boldsymbol{\sigma}_{h}\right)-\mathcal{L}\left(\boldsymbol{\sigma}_{h}\right) \\
& -\frac{1}{2}\left[\left(\mathbf{u}_{1, h}^{\boldsymbol{\lambda}_{1, h}, \mathbf{f}_{1}}-\mathcal{P}_{1}\left(\mathbf{u}_{2, h}^{\lambda_{2, h}, \mathbf{f}_{2}}\right), \mathbf{u}_{1}^{\sigma_{1, h}}-\mathbf{u}_{2}^{\sigma_{2, h}}\right)_{\frac{1}{2}, \Gamma_{1}}+\left(p_{2, h}^{\lambda_{2, h}, \mathbf{f}_{2}}-\mathcal{P}_{2}\left(p_{1, h}^{\boldsymbol{\lambda}_{1, h}, \mathbf{f}_{1}}\right), p_{2}^{\sigma_{2, h}}-p_{1}^{\sigma_{1, h}}\right)_{\frac{1}{2}, \Gamma_{2}}\right] \\
& =\pi\left(\boldsymbol{\lambda}-\boldsymbol{\eta}_{h}, \boldsymbol{\sigma}_{h}\right)+\frac{1}{2}\left(\mathbf{u}_{1}^{\boldsymbol{\lambda}_{1, h}}-\mathbf{u}_{2}^{\lambda_{2, h}}, \mathbf{u}_{1}^{\boldsymbol{\sigma}_{1, h}}-\mathbf{u}_{2}^{\sigma_{2, h}}\right)_{\frac{1}{2}, \Gamma_{1}} \\
& +\frac{1}{2}\left(p_{2}^{\lambda_{2, h}}-p_{1}^{\boldsymbol{\lambda}_{1, h}}, p_{2}^{\sigma_{2, h}}-p_{1}^{\boldsymbol{\sigma}_{1, h}}\right)_{\frac{1}{2}, \Gamma_{2}} \\
& +\frac{1}{2}\left(\mathbf{u}_{1}^{0, \mathbf{f}_{1}}-\mathbf{u}_{2}^{0, \mathbf{f}_{2}}, \mathbf{u}_{1}^{\boldsymbol{\sigma}_{1, h}}-\mathbf{u}_{2}^{\sigma_{2, h}}\right)_{\frac{1}{2}, \Gamma_{1}}+\frac{1}{2}\left(p_{2}^{0, \mathbf{f}_{2}}-p_{1}^{0, \mathbf{f}_{1}}, p_{2}^{\sigma_{2, h}}-p_{1}^{\boldsymbol{\sigma}_{1, h}}\right)_{\frac{1}{2}, \Gamma_{2}} \\
& -\frac{1}{2}\left[\left(\mathbf{u}_{1, h}^{\boldsymbol{\lambda}_{1, h}, \mathbf{f}_{1}}-\mathcal{P}_{1}\left(\mathbf{u}_{2, h}^{\lambda_{2, h}, \mathbf{f}_{2}}\right), \mathbf{u}_{1}^{\boldsymbol{\sigma}_{1, h}}-\mathbf{u}_{2}^{\sigma_{2, h}}\right)_{\frac{1}{2}, \Gamma_{1}}+\left(p_{2, h}^{\lambda_{2, h}, \mathbf{f}_{2}}-\mathcal{P}_{2}\left(p_{1, h}^{\boldsymbol{\lambda}_{1, h}, \mathbf{f}_{1}}\right), p_{2}^{\sigma_{2, h}}-p_{1}^{\sigma_{1, h}}\right)_{\frac{1}{2}, \Gamma_{2}}\right] \\
& =\pi\left(\boldsymbol{\lambda}-\boldsymbol{\eta}_{h}, \boldsymbol{\sigma}_{h}\right) \\
& \left.+\frac{1}{2}\left(\left(\mathbf{u}_{1}^{\boldsymbol{\lambda}_{1, h}, \mathbf{f}_{1}}-\mathbf{u}_{2}^{\lambda_{2, h}, \mathbf{f}_{2}}\right)-\left(\mathbf{u}_{1, h}^{\boldsymbol{\lambda}_{1, h}, \mathbf{f}_{1}}-\mathcal{P}_{1}\left(\mathbf{u}_{2, h}^{\lambda_{2, h}, \mathbf{f}_{2}}\right)\right), \mathbf{u}_{1}^{\boldsymbol{\sigma}_{1, h}}-\mathbf{u}_{2}^{\sigma_{2, h}}\right)\right)_{\frac{1}{2}, \Gamma_{1}} \\
& +\frac{1}{2}\left(\left(p_{2}^{\lambda_{2, h}, \mathbf{f}_{2}}-p_{1}^{\boldsymbol{\lambda}_{1, h}, \mathbf{f}_{1}}\right)-\left(p_{2, h}^{\lambda_{2, h}, \mathbf{f}_{2}}-\mathcal{P}_{2}\left(p_{1, h}^{\boldsymbol{\lambda}_{1, h}, \mathbf{f}_{1}}\right)\right), p_{2}^{\sigma_{2, h}}-p_{1}^{\sigma_{1, h}}\right)_{\frac{1}{2}, \Gamma_{2}} \\
& =\pi\left(\boldsymbol{\lambda}-\boldsymbol{\eta}_{h}, \boldsymbol{\sigma}_{h}\right)-\frac{1}{2}\left(\mathbf{u}_{2}^{\lambda_{2, h}, \mathbf{f}_{2}}-\mathcal{P}_{1}\left(\mathbf{u}_{2, h}^{\lambda_{2, h}, \mathbf{f}_{2}}\right), \mathbf{u}_{1}^{\boldsymbol{\sigma}_{1, h}}-\mathbf{u}_{2}^{\sigma_{2, h}}\right)_{\frac{1}{2}, \Gamma_{1}} \\
& -\frac{1}{2}\left(p_{1}^{\boldsymbol{\lambda}_{1, h}, \mathbf{f}_{1}}-\mathcal{P}_{2}\left(p_{1, h}^{\boldsymbol{\lambda}_{1, h}, \mathbf{f}_{1}}\right), p_{2}^{\sigma_{2, h}}-p_{1}^{\boldsymbol{\sigma}_{1, h}}\right)_{\frac{1}{2}, \Gamma_{2}} \\
& \leq\left(\left\|\boldsymbol{\lambda}-\boldsymbol{\eta}_{h}\right\|+\frac{1}{2}\left\|\mathbf{u}_{2}^{\lambda_{2, h}, \mathbf{f}_{2}}-\mathcal{P}_{1}\left(\mathbf{u}_{2, h}^{\lambda_{2, h}, \mathbf{f}_{2}}\right)\right\|_{\frac{1}{2}, \Gamma_{1}}+\frac{1}{2}\left\|p_{1}^{\boldsymbol{\lambda}_{1, h}, \mathbf{f}_{1}}-\mathcal{P}_{2}\left(p_{1, h}^{\boldsymbol{\lambda}_{1, h}, \mathbf{f}_{1}}\right)\right\|_{\frac{1}{2}, \Gamma_{2}}\right)\left\|\boldsymbol{\sigma}_{h}\right\| .
\end{aligned}
$$

Then, in view of the triangular inequality, it holds for any $\boldsymbol{\eta}_{h} \in \boldsymbol{\Lambda}_{h}$

$$
\begin{aligned}
\| \boldsymbol{\lambda}- & \boldsymbol{\lambda}_{h}\|\leq\| \boldsymbol{\lambda}-\boldsymbol{\eta}_{h}\|+\| \boldsymbol{\sigma}_{h} \| \\
& \leq 2\left\|\boldsymbol{\lambda}-\boldsymbol{\eta}_{h}\right\|+\frac{1}{2}\left\|\mathbf{u}_{2}^{\lambda_{2, h}, \mathbf{f}_{2}}-\mathcal{P}_{1}\left(\mathbf{u}_{2, h}^{\lambda_{2, h}, \mathbf{f}_{2}}\right)\right\|_{\frac{1}{2}, \Gamma_{1}}+\frac{1}{2}\left\|p_{1}^{\boldsymbol{\lambda}_{1, h}, \mathbf{f}_{1}}-\mathcal{P}_{2}\left(p_{1, h}^{\boldsymbol{\lambda}_{1, h}, \mathbf{f}_{1}}\right)\right\|_{\frac{1}{2}, \Gamma_{2}} \\
& \leq 2\left\|\boldsymbol{\lambda}-\boldsymbol{\eta}_{h}\right\|+\sup _{\eta_{2, h} \in \Lambda_{2, h}}\left\|\mathbf{u}_{2}^{\eta_{2, h}, \mathbf{f}_{2}}-\mathcal{P}_{1}\left(\mathbf{u}_{2, h}^{\eta_{2, h}, \mathbf{f}_{2}}\right)\right\|_{\frac{1}{2}, \Gamma_{1}}+\sup _{\boldsymbol{\eta}_{1, h} \in \boldsymbol{\Lambda}_{1, h}}\left\|p_{1}^{\boldsymbol{\eta}_{1, h}, \mathbf{f}_{1}}-\mathcal{P}_{2}\left(p_{1, h}^{\boldsymbol{\eta}_{1, h}, \mathbf{f}_{1}}\right)\right\|_{\frac{1}{2}, \Gamma_{2}} .
\end{aligned}
$$

By Lemma 3.3, the first contribution in the last inequality becomes

$$
\begin{aligned}
\| \boldsymbol{\lambda}- & \boldsymbol{\eta}_{h}\left\|^{2}=\frac{1}{2}\right\| \mathbf{u}_{1}^{\boldsymbol{\lambda}_{1}-\boldsymbol{\eta}_{1, h}}-\mathbf{u}_{2}^{\lambda_{2}-\eta_{2, h}}\left\|_{\frac{1}{2}, \Gamma_{1}}^{2}+\frac{1}{2}\right\| p_{2}^{\lambda_{2}-\eta_{2, h}}-p_{1}^{\boldsymbol{\lambda}_{1}-\boldsymbol{\eta}_{1, h}} \|_{\frac{1}{2}, \Gamma_{2}}^{2} \\
\leq & {\left[\left\|\mathbf{u}_{1}^{\boldsymbol{\lambda}_{1}-\boldsymbol{\eta}_{1, h}}\right\|_{\frac{1}{2}, \Gamma_{1}}^{2}+\left\|\mathbf{u}_{2}^{\lambda_{2}-\eta_{2, h}}\right\|_{\frac{1}{2}, \Gamma_{1}}^{2}+\left\|p_{2}^{\lambda_{2}-\eta_{2, h}}\right\|_{\frac{1}{2}, \Gamma_{2}}^{2}+\left\|p_{1}^{\boldsymbol{\lambda}_{1}-\boldsymbol{\eta}_{1, h}}\right\|_{\frac{1}{2}, \Gamma_{2}}^{2}\right] } \\
\leq & {\left[\left(1+\mu^{2} C_{1}^{2} C_{3}^{2}\right)\left\|\boldsymbol{\lambda}_{1}-\boldsymbol{\eta}_{1, h}\right\|_{\frac{1}{2}, \Gamma_{1}}^{2}+\left(1+\|\boldsymbol{\alpha}\|_{C^{1}}^{2} C_{2}^{2} C_{4}^{2}\right)\left\|\lambda_{2}-\eta_{2, h}\right\|_{\frac{1}{2}, \Gamma_{2}}^{2}\right] } \\
\leq & \widetilde{C}^{2}\left[\left\|\boldsymbol{\lambda}_{1}-\boldsymbol{\eta}_{1, h}\right\|_{\frac{1}{2}, \Gamma_{1}}+\left\|\lambda_{2}-\eta_{2, h}\right\|_{\frac{1}{2}, \Gamma_{2}}\right]^{2} .
\end{aligned}
$$

The thesis follows from the arbitrarity of $\boldsymbol{\eta}_{h}$.

4.2. Optimality system. The following optimality system (OS) is associated with the Euler-Lagrange equation (4.10): find the state solutions $\left(\mathbf{u}_{i, h}, p_{i, h}\right) \in \mathbf{V}_{i, h} \times$ $Q_{i, h}$, the auxiliary solutions $\left(\mathbf{w}_{i, h}, q_{i, h}\right) \in \mathbf{V}_{i, h} \times Q_{i, h}$ and the control variable $\boldsymbol{\lambda}_{h}=$ 
$\left(\boldsymbol{\lambda}_{1, h}, \lambda_{2, h}\right) \in \boldsymbol{\Lambda}_{1, h} \times \Lambda_{2, h}$ satisfying

State Problems: find $\mathbf{u}_{1,0, h} \in \mathbf{V}_{1, h}^{0}, p_{1, h} \in Q_{1, h}$ and $\mathbf{u}_{2, h} \in \mathbf{V}_{2, h}, p_{2, h} \in Q_{2, h}$ :

$\left\{\begin{array}{rr}\mathcal{A}_{1, h}\left(\mathbf{u}_{1,0, h}, p_{1, h} ; \mathbf{v}_{1, h}, q_{1, h}\right)=-\mathcal{B}_{1, h}\left(E_{1, h} \boldsymbol{\lambda}_{1, h} ; \mathbf{v}_{1, h}, q_{1, h}\right)+\mathcal{F}_{1, h}\left(\mathbf{v}_{1, h}, q_{1, h}\right) \\ \\ \mathbf{u}_{1, h}=\mathbf{u}_{1,0, h}+E_{1, h} \boldsymbol{\lambda}_{1, h}, & \forall\left(\mathbf{v}_{1, h}, q_{1, h}\right) \in \mathbf{V}_{1, h}^{0} \times Q_{1, h}\end{array}\right.$

$$
\begin{array}{r}
\mathcal{A}_{2, h}\left(\mathbf{u}_{2, h}, p_{2, h} ; \mathbf{v}_{2, h}, q_{2, h}\right)=-\mathcal{B}_{2, h}\left(\lambda_{2, h} ; \mathbf{v}_{2, h}\right)+\mathcal{F}_{2, h}\left(\mathbf{v}_{2, h}, q_{2, h}\right) \\
\forall\left(\mathbf{v}_{2, h}, q_{2, h}\right) \in \mathbf{V}_{2, h} \times Q_{2, h} ;
\end{array}
$$

Auxiliary Problems: find $\mathbf{w}_{1,0, h} \in \mathbf{V}_{1, h}^{0}, s_{1, h} \in Q_{1, h}$ and $\mathbf{w}_{2, h} \in \mathbf{V}_{2, h}, s_{2, h} \in Q_{2, h}$ :

$$
\begin{aligned}
& \left\{\begin{array}{lr}
\mathcal{A}_{1, h}\left(\mathbf{w}_{1,0, h}, s_{1, h} ; \mathbf{v}_{1, h}, q_{1, h}\right)=-\mathcal{B}_{1, h}\left(E_{1, h}\left(\left(\mathbf{u}_{1, h}-\mathcal{P}_{1}\left(\mathbf{u}_{2, h}\right)\right) ; \mathbf{v}_{1, h}, q_{1, h}\right)\right. \\
\mathbf{w}_{1, h}=\mathbf{w}_{1,0, h}+E_{1, h}\left(\left(\mathbf{u}_{1, h}-\mathcal{P}_{1}\left(\mathbf{u}_{2, h}\right)\right),\right. & \forall\left(\mathbf{v}_{1, h}, q_{1, h}\right) \in \mathbf{V}_{1, h}^{0} \times Q_{1, h}
\end{array}\right. \\
& \mathcal{A}_{2, h}\left(\mathbf{w}_{2, h}, s_{2, h} ; \mathbf{v}_{2, h}, q_{2, h}\right)=-\mathcal{B}_{2, h}\left(\left(p_{2, h}-\mathcal{P}_{2}\left(p_{1, h}\right) ; \mathbf{v}_{2, h}\right)\right. \\
& \forall\left(\mathbf{v}_{2, h}, q_{2, h}\right) \in \mathbf{V}_{2, h} \times Q_{2, h} ;
\end{aligned}
$$

Interface Equations:

$$
\begin{array}{ll}
\int_{\Gamma_{1}}\left(\mathbf{u}_{1, h}-\mathcal{P}_{1}\left(\mathbf{u}_{2, h}-\mathbf{w}_{2, h}\right)\right) \boldsymbol{\eta}_{1, h}=0, & \forall \boldsymbol{\eta}_{1, h} \in \boldsymbol{\Lambda}_{1, h} \\
\int_{\Gamma_{2}}^{1}\left(p_{2, h}-\mathcal{P}_{2}\left(p_{1, h}-s_{1, h}\right)\right) \eta_{2, h}=0, & \forall \eta_{2, h} \in \Lambda_{2, h} .
\end{array}
$$

REMARK 4.1. In view of the strong conditions (4.14), for computational convenience in (4.19) we use the $L^{2}\left(\Gamma_{i}\right)$ inner products instead of the $H^{1 / 2}\left(\Gamma_{i}\right)$ ones.

THEOREM 4.5. The optimality system (OS) (4.15)-(4.19) has a unique solution whose control component $\boldsymbol{\lambda}_{h}$ coincides with the unique solution of (4.10).

Proof. Existence. First we notice that (4.15)-(4.16) coincide with (4.4). Let $\boldsymbol{\lambda}_{h}$ be the solution of (4.10), then the associated states $\left(\mathbf{u}_{1, h}^{\boldsymbol{\lambda}_{1, h}, \mathbf{f}_{1}}, p_{1, h}^{\boldsymbol{\lambda}_{1, h}, \mathbf{f}_{1}}\right)$ and $\left(\mathbf{u}_{2, h}^{\lambda_{2, h}, \mathbf{f}_{2}}, p_{2, h}^{\lambda_{2, h}, \mathbf{f}_{2}}\right)$ satisfy (4.15) and (4.16), respectively. In view of Theorem 4.3, the solutions $\left(\mathbf{w}_{1, h}, s_{1, h}\right)$ and $\left(\mathbf{w}_{2, h}, s_{2, h}\right)$ are null and (4.19) are satisfied. Then there exists at least one solution of (OS).

Uniqueness. In view of the linearity of both the Stokes and the Darcy equations, it suffices to prove that, if $\mathbf{f}_{1}=\mathbf{0}, \mathbf{f}_{2}=\mathbf{0}$, then the solution of (4.15)-(4.19) is the null one. From (4.19) it follows that $\left(\mathbf{u}_{1, h}-\mathcal{P}_{1}\left(\mathbf{u}_{2, h}\right)\right)=-\mathcal{P}_{1}\left(\mathbf{w}_{2, h}\right)$ on $\Gamma_{1}$ and $\left(p_{2, h}-\mathcal{P}_{2}\left(p_{1, h}\right)\right)=-\mathcal{P}_{2}\left(s_{1, h}\right)$ on $\Gamma_{2}$, therefore, by the same arguments used in the proof of Lemma 4.1, the auxiliary system (4.17)-(4.18) has the unique solution $\mathbf{w}_{i, h}=\mathbf{0}$ and $s_{i, h}=0$ in $\bar{\Omega}_{i}$, for $=1,2$. Then, by (4.19), we have $\mathbf{u}_{1, h}=\mathcal{P}_{1}\left(\mathbf{u}_{2, h}\right)$ on $\Gamma_{1}$ and $p_{2, h}=\mathcal{P}_{2}\left(p_{1, h}\right)$ on $\Gamma_{2}$. Again proceeding as before, it follows $\mathbf{u}_{i, h}=\mathbf{0}$ and $p_{i, h}=0$ in $\bar{\Omega}_{i}$, and then $\boldsymbol{\lambda}_{1, h}=\mathbf{0}$ and $\lambda_{2, h}=0$.

4.3. Algebraic formulation of the optimality system. System (OS) (4.15)(4.19) has ten unknown functions: the primal state variables $\mathbf{u}_{i, h}$ and $p_{i, h}$, the dual state variables $\mathbf{w}_{i, h}$ and $s_{i, h}$, for $i=1,2$, and the control variables $\boldsymbol{\lambda}_{1, h}, \lambda_{2, h}$. 
Let us introduce the following arrays:

$$
\begin{array}{r}
\underline{\mathbf{u}}_{1}=\left[\mathbf{u}_{1, h}^{0}\left(\mathbf{x}_{j}\right)\right], \quad \underline{p}_{1}=\left[p_{1, h}\left(\mathbf{x}_{j}\right)\right], \quad \underline{\mathbf{w}}_{1}=\left[\mathbf{w}_{1, h}^{0}\left(\mathbf{x}_{j}\right)\right], \quad \underline{s}_{1}=\left[s_{1, h}\left(\mathbf{x}_{j}\right)\right], \\
\underline{\mathbf{u}}_{2}=\left[\mathbf{u}_{2, h}\left(\mathbf{x}_{j}\right)\right], \quad \underline{p}_{2}=\left[p_{2, h}\left(\mathbf{x}_{j}\right)\right], \quad \underline{\mathbf{w}}_{2}=\left[\mathbf{w}_{2, h}\left(\mathbf{x}_{j}\right)\right], \quad \underline{s}_{2}=\left[s_{2, h}\left(\mathbf{x}_{j}\right)\right], \\
\underline{\boldsymbol{\lambda}}_{1}=\left[\boldsymbol{\lambda}_{1, h}\left(\mathbf{x}_{j}\right)\right], \quad \underline{\lambda}_{2}=\left[\lambda_{2, h}\left(\mathbf{x}_{j}\right)\right], \quad \underline{\mathbf{f}}_{i}=\left[\mathcal{F}_{i, h}\left(\boldsymbol{\varphi}_{i, \ell}, \psi_{i, \ell}\right)\right],
\end{array}
$$

while $\mathrm{A}_{i}, \mathrm{~B}_{i}$, and $\mathrm{P}_{i}$ are the matrices defined in Lemma 4.1.

Let $\mathrm{M}_{i}^{\Gamma_{i}}$ be the $(d-1)$-dimensional mass matrix associated with the interface $\Gamma_{i}$, and $\mathbf{M}_{i}^{\Gamma_{i}}=\operatorname{diag}(\underbrace{\mathbf{M}_{i}^{\Gamma_{i}}, \ldots, \mathbf{M}_{i}^{\Gamma_{i}}}_{d})$, and set

$$
\begin{aligned}
& \mathrm{A}=\left[\begin{array}{ll}
\mathrm{A}_{1} & 0 \\
0 & \mathrm{~A}_{2}
\end{array}\right], \mathrm{B}=\left[\begin{array}{ll}
\mathrm{B}_{1} & 0 \\
0 & \mathrm{~B}_{2}
\end{array}\right], \mathrm{M}^{\Gamma}=\left[\begin{array}{ll}
\mathbf{M}_{1}^{\Gamma_{1}} & 0 \\
0 & \mathrm{M}_{2}^{\Gamma_{2}}
\end{array}\right], \mathrm{P}=\left[\begin{array}{cc}
0 & \mathrm{P}_{1} \\
\mathrm{P}_{2} & 0
\end{array}\right], \\
& \underline{\mathbf{u}}=\left[\begin{array}{l}
\underline{\mathbf{u}}_{1} \\
\underline{p}_{1} \\
\underline{\mathbf{u}}_{2} \\
\underline{p}_{2}
\end{array}\right], \quad \underline{\mathbf{w}}=\left[\begin{array}{l}
\underline{\underline{s}}_{1} \\
\underline{\mathbf{w}}_{2} \\
\underline{\mathbf{s}}_{2} \\
\underline{s}_{2}
\end{array}\right], \quad \underline{\boldsymbol{\lambda}}=\left[\begin{array}{l}
\underline{\boldsymbol{\lambda}}_{1} \\
\underline{\lambda}_{2}
\end{array}\right], \quad \underline{\mathbf{f}}=\left[\begin{array}{l}
\underline{\mathbf{f}}_{1} \\
\underline{\mathbf{f}}_{2}
\end{array}\right] .
\end{aligned}
$$

The algebraic counterpart of OS (4.15)-(4.19) reads

$$
\left[\begin{array}{ccc}
\mathrm{A} & 0 & \mathrm{~B} \\
-\mathrm{BP} & \mathrm{A} & \mathrm{B} \\
-\mathrm{M}^{\Gamma} \mathrm{P} & \mathrm{M}^{\Gamma} \mathrm{P} & \mathrm{M}^{\Gamma}
\end{array}\right]\left[\begin{array}{l}
\underline{\mathbf{u}} \\
\underline{\mathbf{w}} \\
\underline{\boldsymbol{\lambda}}
\end{array}\right]=\left[\begin{array}{l}
\underline{\mathbf{f}} \\
\mathbf{0} \\
\mathbf{0}
\end{array}\right]
$$

and the Schur-complement system w.r.t. the control variable $\underline{\boldsymbol{\lambda}}$ reads

$$
\mathrm{S} \underline{\boldsymbol{\lambda}}=\underline{\psi}
$$

where $\underline{\boldsymbol{\psi}}=\mathrm{M}^{\Gamma}\left(\mathrm{I}+\left(\mathrm{I}-\mathrm{PA}^{-1} \mathrm{~B}\right) \mathrm{PA}^{-1} \underline{\mathbf{f}}\right.$, and $\mathrm{S}$ is the Schur-complement matrix

$$
S=M^{\Gamma}\left(I-\left[\begin{array}{ll}
-P & P
\end{array}\right]\left[\begin{array}{cc}
A & 0 \\
-B P & A
\end{array}\right]^{-1}\left[\begin{array}{l}
B \\
B
\end{array}\right]\right) .
$$

Since the mass matrix $\mathrm{M}^{\Gamma}$ is not singular, we can scale the system by leftmultiplying the last row of (4.20), or equivalently both sides of $(4.21)$, by $\left(\mathrm{M}^{\Gamma}\right)^{-1}$. This operation can in fact be regarded as (left) preconditioning system (4.21) by the matrix $\mathrm{M}^{\Gamma}$.

The solution of the Schur-complement system (4.21) can be efficiently computed by Krylov methods (specifically, we will use Bi-CGStab [48]). We first compute the right-hand side of (4.21) as in Algorithm 4.1. Then, given the array $\underline{\boldsymbol{\lambda}}^{(k)}$ at the $k$ th iteration of Bi-CGStab, the matrix vector product $\underline{\boldsymbol{\chi}}^{(k)}=\mathrm{S} \underline{\boldsymbol{\lambda}}^{(k)}$ as in Algorithm 4.2.

Algorithm 4.1. Given $\mathbf{f}_{1}$ and $\mathbf{f}_{2}$, compute $\underline{\boldsymbol{\psi}}$.

1. Solve (4.15) and (4.16) using homogeneous Dirichlet data on the interfaces $\Gamma_{1}$ and $\Gamma_{2}$ and right-hand sides $\underline{\mathbf{f}}_{1}$ and $\underline{\mathbf{f}}_{2}$, respectively;

2. solve the dual problems (4.17) and (4.18), store the solutions in $\left(\mathbf{u}_{i}, p_{i}\right)$ and $\left(\mathbf{w}_{i}, s_{i}\right), i=1,2$

3. compute $\underline{\boldsymbol{\psi}}^{(k)}=\left[\begin{array}{l}-\mathrm{P}_{1}\left(\underline{\mathbf{u}}_{2}-\underline{\mathbf{w}}_{2}\right) \\ -\mathrm{P}_{2}\left(\underline{p}_{1}-\underline{s}_{1}\right)\end{array}\right]$. 
Algorithm 4.2. Given $\underline{\boldsymbol{\lambda}}^{(k)}$, compute $\underline{\boldsymbol{\chi}}^{(k)}=\boldsymbol{S} \underline{\boldsymbol{\lambda}}^{(k)}$.

1. Solve (4.15) and (4.16) using $\underline{\boldsymbol{\lambda}}_{1}^{(k)}$ and $\underline{\lambda}_{2}^{(k)}$ as Dirichlet data on the interfaces $\Gamma_{1}$ and $\Gamma_{2}$, and null right hand sides $\underline{\mathbf{f}}_{1}$ and $\underline{\mathbf{f}}_{2}$, respectively;

2. solve the dual problems (4.17) and (4.18), store the solutions in $\left(\mathbf{u}_{i}, p_{i}\right)$ and $\left(\mathbf{w}_{i}, s_{i}\right), i=1,2$;

3. compute $\underline{\boldsymbol{\chi}}^{(k)}=\left[\begin{array}{l}\underline{\boldsymbol{\lambda}}_{1}^{(k)}-\mathrm{P}_{1}\left(\underline{\mathbf{u}}_{2}-\underline{\mathbf{w}}_{2}\right) \\ \underline{\lambda}_{2}^{(k)}-\mathrm{P}_{2}\left(\underline{p}_{1}-\underline{s}_{1}\right)\end{array}\right]$.

5. Numerical results. We consider some $2 \mathrm{D}$ test cases in which the computational domain represents a vertical section of a 3D volume. The coordinates in the plane are $x$ and $z$. For simplicity, we consider matching meshes in $\Omega_{12}$ and the same polynomial degrees for velocity and pressure in the Stokes and Darcy subdomains. Different discretizations can be adopted inside different subdomains and non-matching grids can be designed on the overlap, as done in [15] for the elliptic-elliptic coupling.

Our aim is twofold. On the one hand we want to numerically assess the robustness of ICDD versus the discretization parameters $(h$ and $p)$ as well as versus the overlap thickness $\delta$ (see Test 1 in Section 5.1). The Bi-CGstab method with a given stopping tolerance $\epsilon$ is used to solve the Schur complement system (4.21). On the other hand, we want to analyze the robustness of the ICDD method with respect to variations of the physical properties in the porous medium and to compare numerical solutions obtained by ICDD with those computed by a Sharp Interface (SI) approach based on the interface conditions (see, e.g. [17])

$$
\mathbf{u}_{1} \cdot \mathbf{n}=\mathbf{u}_{2} \cdot \mathbf{n}, \quad-\left(\mathbf{T}_{1}\left(\mathbf{u}_{1}, p_{1}\right) \mathbf{n}\right) \cdot \mathbf{n}=p_{2}, \quad-\left(\mathbf{T}_{1}\left(\mathbf{u}_{1}, p_{1}\right) \mathbf{n}\right) \cdot \boldsymbol{\tau}=\frac{\alpha_{B J} \mu}{\sqrt{\boldsymbol{\tau}^{T} \boldsymbol{\kappa} \boldsymbol{\tau}}} \mathbf{u}_{1} \cdot \boldsymbol{\tau}
$$

assigned on the sharp interface $\Gamma$ between two non-overlapping subdomains (that we continue to name $\Omega_{1}$ and $\Omega_{2}$, even if they not coincide with those of the ICDD approach, see Fig. 2.1, right), $\mathbf{n}$ is the normal unit vector to $\Gamma$ directed outward of $\Omega_{1}$, $\boldsymbol{\tau}$ represents a set of linear independent unit tangential vectors to $\Gamma$ with $\boldsymbol{\tau} \cdot \mathbf{n}=0$, and $\alpha_{B J}$ is a dimensionless coefficient depending on the geometrical characteristics of the porous medium. Condition $(5.1)_{3}$ is the BJS condition $[1,46,29,34,13,45]$ that establishes proportionality between the tangential component of the flux and that of the free velocity. It represents an approximation of the experimental law of Beavers-Joseph (see $[1,32]$ )

$$
-\left(\mathbf{T}_{1}\left(\mathbf{u}_{1}, p_{1}\right) \mathbf{n}\right) \cdot \boldsymbol{\tau}=\frac{\alpha_{B J} \mu}{\sqrt{\boldsymbol{\tau}^{T} \boldsymbol{\kappa} \boldsymbol{\tau}}}\left(\mathbf{u}_{1}-\mathbf{u}_{2}\right) \cdot \boldsymbol{\tau} \quad \text { on } \Gamma .
$$

Note that (5.2) is in fact a coupling condition whereas $(5.1)_{3}$ is not.

From now on, the acronym SI-BJS will identify the sharp interface approach recalled just now, using the three conditions (5.1).

5.1. Test 1. Let us consider the Stokes-Darcy coupling (2.1) with $\boldsymbol{\kappa}=\kappa \mathbf{I}$ and non-homogeneous boundary conditions chosen in such a way that the exact solution reads

$$
\begin{aligned}
& \mathbf{u}_{1}(x, z)=\mathbf{u}_{2}(x, z)=\left[-\frac{\kappa}{\mu} \frac{\pi}{2} e^{\frac{\pi}{2} x} \sin \left(\frac{\pi}{2} z\right) ;-\frac{\kappa}{\mu} \frac{\pi}{2} e^{\frac{\pi}{2} x} \cos \left(\frac{\pi}{2} z\right)\right] \\
& p_{1}(x, z)=p_{2}(x, z)=e^{\frac{\pi}{2} x} \sin \left(\frac{\pi}{2} z\right)
\end{aligned}
$$


TABLE 5.1

Test 1. ICDD iterations vs the mesh size $h$ for different FEM discretizations. The exact solution is given in (5.3). The overlap thickness is fixed $\delta=0.4$.

\begin{tabular}{ccccc}
\hline$h$ & $\begin{array}{c}\text { stabilized } \\
\mathbb{Q}_{1}-\mathbb{Q}_{1}\end{array}$ & $\begin{array}{c}\text { stabilized } \\
\mathbb{P}_{1}-\mathbb{P}_{1}\end{array}$ & $\begin{array}{c}\text { stabilized } \\
\mathbb{P}_{2}-\mathbb{P}_{2}\end{array}$ & $\begin{array}{c}\mathbb{P}_{2}-\mathbb{P}_{1} \\
\text { (Stokes-compatible) }\end{array}$ \\
\hline $1 / 5$ & 8 & 4 & 4 & 4 \\
$1 / 10$ & 8 & 4 & 4 & 4 \\
$1 / 20$ & 7 & 4 & 4 & 4 \\
$1 / 40$ & 7 & 4 & 4 & 4 \\
$1 / 80$ & 7 & 4 & 4 & 4 \\
\hline
\end{tabular}

TABLE 5.2

Test 1. Estimated convergence orders vs the mesh size $h$ for different discretizations. The exact solution is given in (5.3).

\begin{tabular}{ccccc}
\hline Convergence orders for & $e_{u, 1}$ & $e_{p, 1}$ & $e_{u, 2}$ & $e_{p, 2}$ \\
\hline stab. $\mathbb{Q}_{1}-\mathbb{Q}_{1}$ & 1.715 & 1.810 & 1.513 & 1.575 \\
stab. $\mathbb{P}_{1}-\mathbb{P}_{1}$ & 1.002 & 1.553 & 1.086 & 0.999 \\
stab. $\mathbb{P}_{2}-\mathbb{P}_{2}$ & 2.009 & 2.004 & 1.595 & 1.991 \\
$\mathbb{P}_{2}-\mathbb{P}_{1}$ (Stokes compat.) & 2.000 & 1.999 & 1.963 & 1.000 \\
\hline
\end{tabular}

in $\Omega=(0,1) \times(0,2)$ and with $\kappa=1 / 10$ and $\mu=1$. The overlapping subdomains are $\Omega_{1}=(0,1) \times(1-\delta / 2,2)$ and $\Omega_{2}=(0,1) \times(0,1+\delta / 2)$, with $\delta>0$, and the meshes are uniform and structured in both subdomains.

In Tables 5.1-5.2 we report, respectively, the number of ICDD iterations required to solve system (4.21) up to tolerance $\epsilon=10^{-9}$ and the convergence rates with respect to $h$ (in the range $[1 / 80,1 / 5]$ ) relative to the following errors:

$$
\begin{aligned}
& e_{u, 1}=\left\|\mathbf{u}_{1}-\mathbf{u}_{1 h}\right\|_{H^{1}\left(\Omega_{1}\right)}, \quad e_{p, 1}=\left\|p_{1}-p_{1 h}\right\|_{L^{2}\left(\Omega_{1}\right)}, \\
& e_{u, 2}=\left\|\mathbf{u}_{2}-\mathbf{u}_{2 h}\right\|_{L^{2}\left(\Omega_{2}\right)}, \quad e_{p, 2}=\left\|p_{1}-p_{2 h}\right\|_{H^{1}\left(\Omega_{2}\right)} .
\end{aligned}
$$

The results of Table 5.3 refer to discretizations with stabilized $\mathbb{Q}_{p}-\mathbb{Q}_{p}$ elements.

The numerical results of Tables 5.2-5.3 show that the theoretical convergence orders of $h p-\mathrm{FEM}$ are achieved by ICDD solutions, as stated in Theorem 4.4 , thanks also to the equivalence between the norm $\|\cdot\|$ and the canonical norm in $H^{1 / 2}$ stated in Lemma 3.4.

Here, since the exact solution (5.3) is infinitely smooth, algebraic convergence of order $p$ with respect to $h$ and exponential convergence with respect to $p$ are observed for $\mathbb{Q}_{p}-\mathbb{Q}_{p}(p \geq 1)$ in both Stokes and Darcy domains.

In Table 5.4 we report the number of ICDD iterations required to solve system (4.21) up to tolerance $\epsilon=10^{-9}$ versus the overlap thickness $\delta$. Different situations are considered. The results of the first five rows refer to fixed uniform and structured discretizations in either simplexes or quads; those of the second block are obtained again by considering uniform and cartesian grids, but the mesh size $h$ varies and $\delta=h$; those of the last row are obtained by designing a quasi-uniform mesh of quads of maximum size $h=1 / 5$ outside the overlap $\Omega_{12}$, and a strip of flattened quads of height equal to $\delta$ and width equal to $h=1 / 5$ in $\Omega_{12}$. In this example, the quality of the numerical solution is not affected by the choice of the overlap thickness $\delta$, since the test solution does not feature internal layers.

The results of Tables 5.1, 5.3 and 5.4 show that the number of ICDD iterations is 
TABLE 5.3

Test 1. ICDD iterations, infimum of the cost functional $J_{h}$ (4.6) and errors (5.4) vs the polynomial degree $p$ for stabilized $\mathbb{Q}_{p}-\mathbb{Q}_{p}$ discretization in both Stokes and Darcy subdomains. The exact solution is given in (5.3). The overlap thickness is fixed $\delta=0.4=4 h$.

\begin{tabular}{ccccccc}
\hline$p$ & $\# i t$ & inf $J_{h}$ & $e_{u, 1}$ & $e_{p, 1}$ & $e_{u, 2}$ & $e_{p, 2}$ \\
\hline 1 & 6 & $3.851 \mathrm{e}-18$ & $4.9476 \mathrm{e}-02$ & $2.9216 \mathrm{e}-01$ & $7.5562 \mathrm{e}-02$ & $5.9257 \mathrm{e}-02$ \\
2 & 7 & $4.800 \mathrm{e}-21$ & $1.1310 \mathrm{e}-03$ & $2.7244 \mathrm{e}-03$ & $9.5293 \mathrm{e}-03$ & $8.2701 \mathrm{e}-03$ \\
4 & 6 & $6.118 \mathrm{e}-21$ & $1.6159 \mathrm{e}-07$ & $2.1171 \mathrm{e}-07$ & $8.4074 \mathrm{e}-07$ & $8.0511 \mathrm{e}-07$ \\
6 & 6 & $4.934 \mathrm{e}-22$ & $1.9260 \mathrm{e}-11$ & $1.2030 \mathrm{e}-11$ & $1.0879 \mathrm{e}-10$ & $9.8985 \mathrm{e}-11$ \\
\hline
\end{tabular}

TABLE 5.4

Test 1. ICDD iterations versus the overlap thickness $\delta$.

\begin{tabular}{|c|c|c|c|c|c|c|}
\hline fixed $h=0.04$ & $\delta=$ & $5 h$ & $4 h$ & $3 h$ & $2 h$ & $h$ \\
\hline stab. $\mathbb{P}_{1}-\mathbb{P}_{1}$ & $\# i t$ & 6 & 8 & 10 & 14 & 21 \\
\hline stab. $\mathbb{P}_{2}-\mathbb{P}_{2}$ & $\# i t$ & 6 & 8 & 11 & 21 & 28 \\
\hline $\mathbb{P}_{2}-\mathbb{P}_{1}$ (Stokes compat.) & $\# i t$ & 6 & 8 & 9 & 11 & 20 \\
\hline fixed $h=0.04$ & $\delta=$ & $10 h$ & $8 h$ & $6 h$ & $4 h$ & $2 h$ \\
\hline stab. $\mathbb{Q}_{1}-\mathbb{Q}_{1}$ & $\# i t$ & 7 & 7 & 8 & 8 & 9 \\
\hline \multirow[t]{2}{*}{ stab. $\mathbb{Q}_{4}-\mathbb{Q}_{4}$} & $\# i t$ & 5 & 5 & 5 & 6 & 8 \\
\hline & $h=\delta=$ & $1 / 3$ & $1 / 6$ & $1 / 12$ & $1 / 25$ & \\
\hline stab. $\mathbb{P}_{1}-\mathbb{P}_{1}$ & $\# i t$ & 4 & 8 & 14 & 22 & \\
\hline stab. $\mathbb{P}_{2}-\mathbb{P}_{2}$ & $\# i t$ & 5 & 10 & 20 & 41 & \\
\hline \multirow[t]{2}{*}{$\mathbb{P}_{2}-\mathbb{P}_{1}$ (Stokes compat.) } & $\# i t$ & 4 & 6 & 9 & 21 & \\
\hline & $h=\delta=$ & $2 / 9$ & $2 / 17$ & $2 / 33$ & $2 / 65$ & $2 / 129$ \\
\hline stab. $\mathbb{Q}_{1}-\mathbb{Q}_{1}$ & $\# i t$ & 7 & 8 & 9 & 10 & 14 \\
\hline $\begin{array}{l}\text { uniform mesh in } \Omega \backslash \\
\text { stab. } \mathbb{Q}_{4}-\mathbb{Q}_{4}\end{array}$ & $\begin{array}{r}12, h=0 \\
\delta= \\
\# i t\end{array}$ & $\begin{array}{c}\text {. One s } \\
1 . e-2 \\
11\end{array}$ & $\begin{array}{c}\text { ip of qu } \\
2 . e-3 \\
32\end{array}$ & $\begin{array}{c}\text { ds of size } \\
1 . e-3 \\
44\end{array}$ & $\begin{array}{c}5 \times h \text { in } \\
2 . e-4 \\
60\end{array}$ & $\begin{array}{c}12 . e-4 \\
67\end{array}$ \\
\hline
\end{tabular}

independent of the discretization parameters $h$ and $p$, while it depends on the overlap thickness $\delta$; indeed \#it $=\mathcal{O}\left(\delta^{q}\right)$, for some $q$ between $-1 / 2$ and -1 . A similar behavior has been observed when ICDD has been applied to elliptic problems in [15] and to the Stokes equations in [16].

5.2. Test 2: Reproducing the Beavers-Joseph experiment. This simple test case mimics the experiment presented by Beavers and Joseph in their seminal paper [1]. We consider the rectilinear flow of a viscous fluid through a $2 \mathrm{D}$ parallel channel formed by an impermeable upper wall and a permeable lower wall. The latter defines a nominal surface for the permeable material. A uniform pressure gradient is maintained in the longitudinal direction in both the channel and the permeable media. Following the classification introduced by Levy and Sanchez-Palencia [35], this test involves "near parallel flows", i.e. flows for which $\nabla p$ in the porous domain is parallel to the nominal interface and the velocity inside the porous domain is much smaller than in the fluid domain.

We set $\Omega=(0,0.25) \times(-0.075,0)$ (in meters). Given the overlap thickness $\delta>0$ and $z_{\Gamma}=-0.055$, the overlapping subdomains are $\Omega_{1}=(0,0.25) \times\left(z_{\Gamma}-\delta, 0\right)$ and $\Omega_{2}=(0,0.25) \times\left(-0.075, z_{\Gamma}\right)$, so that $\Gamma_{1}=(0,0.25) \times\left\{z_{\Gamma}-\delta\right\}, \Gamma_{2}=(0,0.25) \times\left\{z_{\Gamma}\right\}$, and the overlap is thought to be embedded in the Darcy domain. The fluid is water 
with density $\rho=10^{3}\left[\mathrm{~kg} / \mathrm{m}^{3}\right]$ and dynamic viscosity $\mu=10^{-3}[\mathrm{~kg} /(\mathrm{m} \mathrm{s})]$. The porous medium is characterized by its intrinsic permeability $\boldsymbol{\kappa}\left[\mathrm{m}^{2}\right]$, that we suppose homogeneous and isotropic, i.e. $\boldsymbol{\kappa}=\kappa \mathbf{I}$, where $\kappa>0$ and $\mathbf{I}$ is the identity tensor. To guarantee a uniform pressure gradient we set $\mathbf{f}_{1}=(1,0)^{t}\left[\mathrm{~kg} /\left(\mathrm{m}^{2} \mathrm{~s}^{2}\right)\right]$, we impose a parabolic inflow on the left-hand side: $\mathbf{u}_{1}=\left(-10^{3} z(z+0.055), 0\right)^{t}[\mathrm{~m} / \mathrm{s}]$ if $-0.055 \leq$ $z \leq 0, \mathbf{u}_{1}=(0,0)^{t}[\mathrm{~m} / \mathrm{s}]$ otherwise, and $\mathbf{T}_{1}\left(\mathbf{u}_{1}, p_{1}\right) \cdot \mathbf{n}=(0,(-2 z-0.055))^{t}\left[\mathrm{~kg} /\left(\mathrm{m} \mathrm{s}^{2}\right)\right]$ on the right-hand side. In the Darcy domain we impose an external force $\mathbf{f}_{2}=\mathbf{0}$ and the following boundary data: $\mathbf{u}_{2} \cdot \mathbf{n}_{2}=0$ on the bottom horizontal side, $p_{2}=0.25$ $\left[\mathrm{kg} /\left(\mathrm{ms}^{2}\right)\right]$ on the left vertical side $\{0\} \times(-0.075,-0.055)$ and $p_{2}=0$ on the right one $\{0.25\} \times(-0.075,-0.055)$.

The choice of $\delta$ affects the Stokes solution computed by the ICDD method. At the same time it is known (see, e.g., $[46,40,30]$ ) that an internal layer of thickness $\mathcal{O}(\sqrt{\kappa})=\mathcal{O}(\varepsilon)$ occurs between the fluid and the porous domains. We therefore set $\delta=c \sqrt{\kappa}$, where $c>0$ is a suitable constant, that may depend on the geometry of the porous medium. With this choice, the minimization of $\left\|\mathbf{u}_{1}-\mathbf{u}_{2}\right\|$ on $\Gamma_{1}$ imposed by the ICDD method not only implies the continuity of the normal component of the velocity $(5.1)_{1}$ on $\Gamma_{1}$, but also the fulfillment of a Beavers-Joseph-like condition (5.5). In fact, let us set $\mathbf{u}_{i}=\left(u_{i}, v_{i}\right)^{t}$, for $i=1,2$. In this case condition (5.2) reduces to

$$
\frac{\partial u_{1}}{\partial z}\left(z_{\Gamma}^{+}\right)=\frac{\alpha_{B J}}{\sqrt{\kappa}}\left(u_{1}\left(z_{\Gamma}^{+}\right)-u_{2}\left(z_{\Gamma}^{-}\right)\right),
$$

where $z_{\Gamma}^{+}$and $z_{\Gamma}^{-}$are suitable points close to $z_{\Gamma}$ such that $z_{\Gamma}^{+} \in \Omega_{1}$ and $z_{\Gamma}^{-} \in \Omega_{2}$.

Now we approximate the derivative on the left of (5.5) by a first-order finite difference scheme with step size $\delta=z_{\Gamma}^{+}-z_{\Gamma}^{-}$:

$$
\frac{u_{1}\left(z_{\Gamma}^{+}\right)-u_{1}\left(z_{\Gamma}^{-}\right)}{\delta} \simeq \frac{\alpha_{B J}}{\sqrt{\kappa}}\left(u_{1}\left(z_{\Gamma}^{+}\right)-u_{2}\left(z_{\Gamma}^{-}\right)\right),
$$

and we choose

$$
\delta=\frac{\sqrt{\kappa}}{\alpha_{B J}} .
$$

By looking at $z_{\Gamma}^{+}$(respectively, $z_{\Gamma}^{-}$) as the vertical coordinate of the interface $\Gamma_{2}$ (respectively, $\left.\Gamma_{1}\right)$ and by using $(5.7)$, we conclude that the interface condition $u_{1}\left(z_{\Gamma}^{-}\right)=$ $u_{2}\left(z_{\Gamma}^{-}\right)$(that we impose in the ICDD method) can be regarded as an approximation of the Beavers-Joseph condition (5.5).

The idea of using an interfacial region of thickness $\delta \simeq \sqrt{\kappa} / \alpha_{O W}$ has been considered in [40,41], where $\alpha_{O W}$ is a suitable constant depending on the porous medium. However, in that case the authors solve a Stokes problem with an additional term featuring a variable porosity in such a region, a Darcy model with Brinkman correction in the porous domain and impose (for this test case) the continuity of both the tangential velocity and its normal derivative at the interfaces. As a matter of fact, ICDD forces the continuity of both components of the velocity on the lower interface $\Gamma_{1}$ and the continuity of the pressure on the upper one $\Gamma_{2}$, but the Stokes and the Darcy solutions may not coincide on the overlap. The parameter $\alpha_{B J}$ depends on the geometry of the porous material. Its characterization is beyond the aim of this paper; for simplicity we consider $\alpha_{B J}=1$.

After setting $\delta=\sqrt{\kappa}=\varepsilon$, we compute numerical solutions of this test case for $\kappa=10^{-6}, 10^{-8}, 10^{-10}$. Larger values of $\kappa$ would lead to a meaningless coupling since 

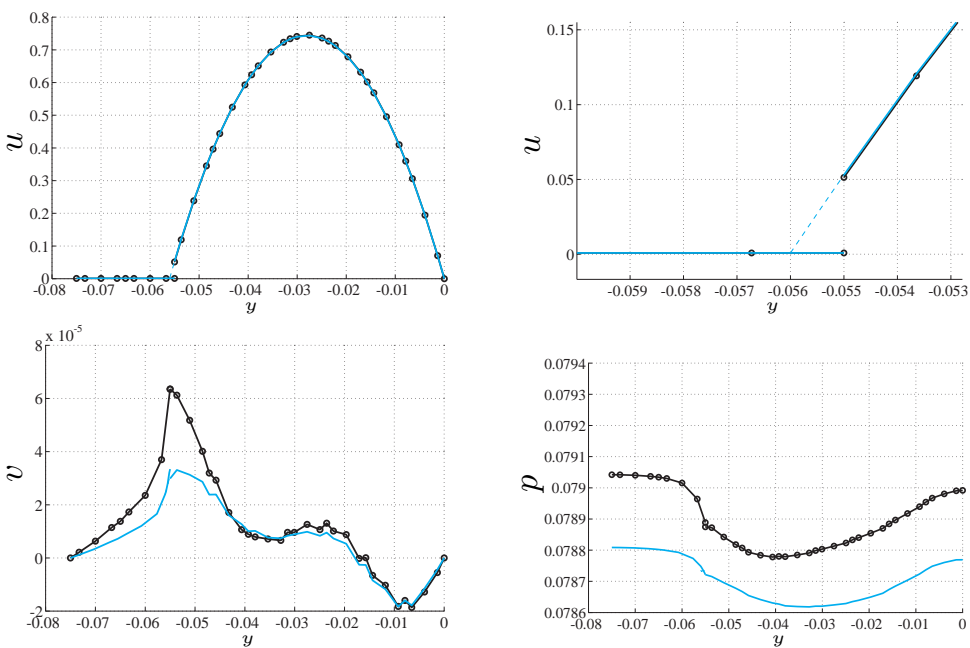

FIG. 5.1. Test 2. Profiles of $u$ (top), $v$ (bottom left), and $p$ (bottom right) at $x=0.15625$ for $\boldsymbol{\kappa}=10^{-6} \mathbf{I}$ and SI-BJS approach (black line), ICDD with $\delta=10^{-3}$ (light blue). The top-right picture is a zoom of the top-left one around the sharp interface $z_{\Gamma}$. The dashed line represents the first component of the Stokes velocity in the overlap region.

TABLE 5.5

Test 2. Relative maximum norm of the distance between SI-BJS and ICDD solutions at the coordinate $\bar{x}=0.15625 . e_{u}(\bar{x})=\left\|u_{S I-B J S}-u_{I C D D}\right\|_{L^{\infty}(I)} /\left\|u_{S I-B J S}\right\|_{L^{\infty}(I)}, e_{v}(\bar{x})=\| v_{S I-B J S}-$ $v_{I C D D}\left\|_{L^{\infty}(I)} /\right\| v_{S I-B J S}\left\|_{L^{\infty}(I)}, e_{p}(\bar{x})=\right\| p_{S I-B J S}-p_{I C D D}\left\|_{L^{\infty}(I)} /\right\| p_{S I-B J S} \|_{L \infty(I)}$, where $I=\{\bar{x}\} \times(-0.075,-0.055)$ in the Darcy domain and $I=\{\bar{x}\} \times(-0.055,0)$ in the Stokes domain

\begin{tabular}{ccccccc}
\hline$\kappa$ & \multicolumn{3}{c}{ Stokes domain } & \multicolumn{3}{c}{ Darcy domain } \\
& $e_{u}(\bar{x})$ & $e_{v}(\bar{x})$ & $e_{p}(\bar{x})$ & $e_{u}(\bar{x})$ & $e_{v}(\bar{x})$ & $e_{p}(\bar{x})$ \\
\hline $10^{-6}$ & $2.277 \mathrm{e}-03$ & $5.339 \mathrm{e}-01$ & $2.870 \mathrm{e}-03$ & $2.868 \mathrm{e}-02$ & $4.936 \mathrm{e}-01$ & $3.014 \mathrm{e}-03$ \\
$10^{-8}$ & $2.628 \mathrm{e}-05$ & $7.469 \mathrm{e}-03$ & $1.154 \mathrm{e}-04$ & $4.884 \mathrm{e}-02$ & $4.314 \mathrm{e}-01$ & $1.111 \mathrm{e}-04$ \\
$10^{-10}$ & $1.058 \mathrm{e}-05$ & $1.364 \mathrm{e}-03$ & $1.478 \mathrm{e}-05$ & $4.920 \mathrm{e}-02$ & $4.224 \mathrm{e}-01$ & $3.597 \mathrm{e}-05$ \\
\hline
\end{tabular}

the characteristic length of the pores would become comparable with the characteristic length $x_{s}=0.005$ of the Stokes domain (about the height of the channel). The solution of this problem is quite independent of the $x$ variable, so we analyze its behavior at the fixed abscissa $\bar{x}=0.15625$.

In Figure 5.1 we show the SI-BJS and ICDD solutions computed at $\bar{x}$ with $\kappa=$ $10^{-6}\left[\mathrm{~m}^{2}\right]$. The SI-BJS solution is obtained by decomposing $\Omega$ with nominal interface in $z_{\Gamma}=-0.055$, while the ICDD solution is found by setting $z_{\Gamma_{1}}=z_{\Gamma}-\delta, z_{\Gamma_{2}}=z_{\Gamma}$, and overlap thickness $\delta=\varepsilon=10^{-3}$ (computed by (5.7)).

For smaller values of $\kappa$, the differences between the SI-BJS and ICDD solutions are reported in Table 5.5, where $e_{u}(\bar{x}), e_{v}(\bar{x})$ and $e_{p}(\bar{x})$ are the relative difference in $L^{\infty}$-norm between the SI-BJS and ICDD solutions at $\bar{x}=0.15625$, for $z<z_{\Gamma}$ (Darcy domain for sharp interface approach) and $z>z_{\Gamma}$ (Stokes domain for sharp interface approach). The results of Table 5.5 show that, when $\delta$ is chosen as in (5.7), the ICDD and SI-BJS solutions match very well. In all these cases, the results in Table 5.6 confirm the theoretical analysis in [1], [46], and [30], for which the order of magnitude of both components of the Darcy velocity is about $\mathcal{O}(\kappa)=\mathcal{O}\left(\varepsilon^{2}\right)$, while the tangential component of the Stokes velocity at the interface behaves like $\mathcal{O}(\sqrt{\kappa})$ when $\kappa \rightarrow 0$. 
TABLE 5.6

Test 2. Values of the first $\left(u_{i}, i=1,2\right)$ and second $\left(v_{i}, i=1,2\right)$ component of the velocity at $\left(\bar{x}, z_{\Gamma}\right)$ with $\bar{x}=0.15625$ and $z_{\Gamma}=z_{\Gamma_{2}} . v_{1} \neq v_{2}$ since ICDD imposes continuity of $v$ on the interface $\Gamma_{1}$. Values of $v_{2}$ of the ICDD solution match well the corresponding values of the SI-BJS solution.

\begin{tabular}{c|cccc|ccc}
\hline$\kappa$ & \multicolumn{4}{|c|}{ ICDD solution } & \multicolumn{3}{c}{ SI-BJS solution } \\
& $u_{1}$ & $u_{2}$ & $v_{1}$ & $v_{2}$ & $u_{1}$ & $u_{2}$ & $v_{1}=v_{2}$ \\
\hline $10^{-6}$ & $5.30 \mathrm{e}-02$ & $8.95 \mathrm{e}-04$ & $2.94 \mathrm{e}-05$ & $3.20 \mathrm{e}-05$ & $5.13 \mathrm{e}-02$ & $8.70 \mathrm{e}-04$ & $6.32 \mathrm{e}-05$ \\
$10^{-8}$ & $5.48 \mathrm{e}-03$ & $9.89 \mathrm{e}-06$ & $3.29 \mathrm{e}-07$ & $1.45 \mathrm{e}-07$ & $5.46 \mathrm{e}-03$ & $9.40 \mathrm{e}-06$ & $1.85 \mathrm{e}-07$ \\
$10^{-10}$ & $5.50 \mathrm{e}-04$ & $9.98 \mathrm{e}-08$ & $2.36 \mathrm{e}-08$ & $1.65 \mathrm{e}-09$ & $5.50 \mathrm{e}-04$ & $9.49 \mathrm{e}-08$ & $1.95 \mathrm{e}-09$ \\
$10^{-12}$ & $5.50 \mathrm{e}-05$ & $1.01 \mathrm{e}-09$ & $2.06 \mathrm{e}-09$ & $1.30 \mathrm{e}-11$ & $5.50 \mathrm{e}-05$ & $9.50 \mathrm{e}-10$ & $1.98 \mathrm{e}-11$ \\
\hline
\end{tabular}
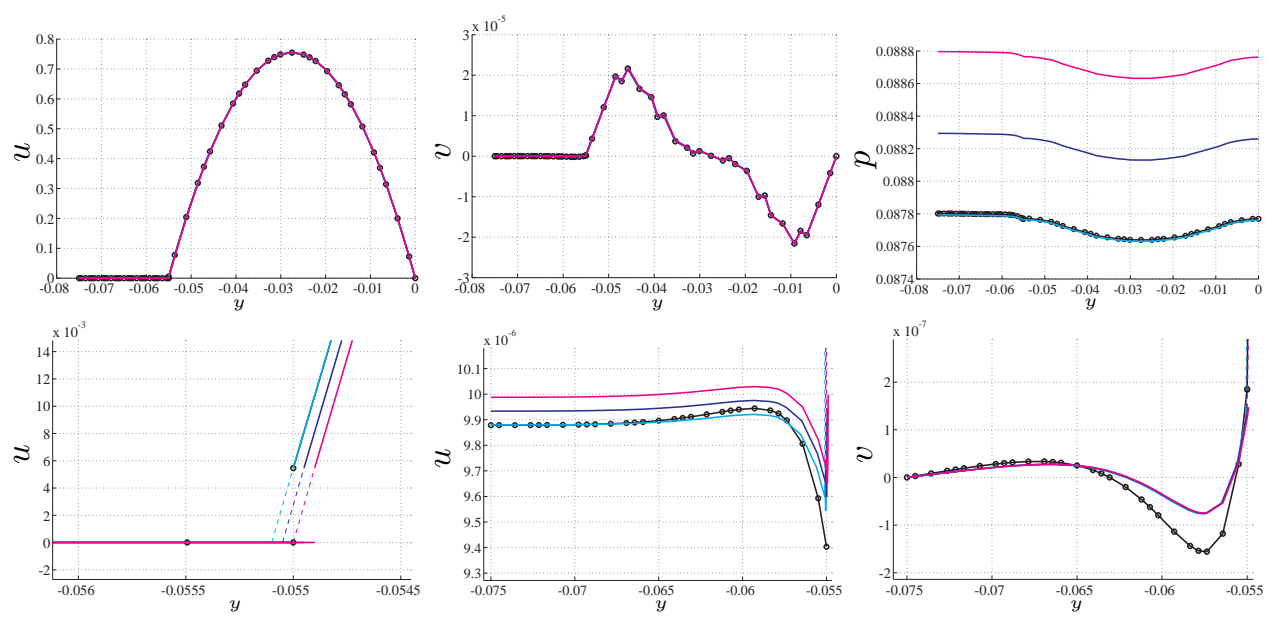

FIG. 5.2. Test 2. Profiles of $u$, $v$, and $p$ (top) at $\bar{x}=0.15625$ for $\boldsymbol{\kappa}=10^{-8} \mathbf{I}$, computed by SI-BJS (black line) and ICDD (colored) with $\delta=10^{-4}$ : lower overlap (light blue), medium overlap (dark blue), and upper overlap (magenta). The bottom left and middle pictures are zooms of the first component of the velocity around the interface and inside the Darcy domain. The bottom-right one is a zoom of the second component of the velocity in the porous domain. Dashed lines inside the overlap refer to the Stokes solutions.

We fix now the permeability $\kappa=10^{-8}\left[\mathrm{~m}^{2}\right]$ and the thickness overlap $\delta=10^{-4}$, but we consider three decompositions which differ one another for the position of the overlap with respect to the position $z_{\Gamma}$ of the nominal interface $\Gamma$ of SI-BJS:

- lower overlap, for which $z_{\Gamma_{1}}=z_{\Gamma}-\delta$ and $z_{\Gamma_{2}}=z_{\Gamma}$;

- medium overlap, for which $z_{\Gamma_{1}}=z_{\Gamma}-\delta / 2$ and $z_{\Gamma_{2}}=z_{\Gamma}+\delta / 2$;

- upper overlap, for which $z_{\Gamma_{1}}=z_{\Gamma}$ and $z_{\Gamma_{2}}=z_{\Gamma}+\delta$.

In Figure 5.2 we show the corresponding solutions at $\bar{x}=0.15625$, while in Table 5.7 we show the relative errors between the ICDD and SI-BJS solutions in the $L^{\infty}$ norm.

The ICDD solution obtained with the lower overlap is the closest to the SI-BJS solution, however, differences among ICDD solutions with different overlaps are very small.

5.3. Test 3: Cross-flow membrane filtration. This case addresses the coupling of Stokes and Darcy flows in a cross-flow membrane filtration setting. The data for this test case are taken from [24]. The domain is $\Omega=(0,0.015) \times(-0.0075,0)$ (in meters). Given the overlap thickness $\delta>0$ and $z_{\Gamma}=-0.0055$, the overlapping 
TABLE 5.7

Test 2. Relative maximum norm of the distance between the SI-BJS and ICDD solutions on the cut $\bar{x}=0.15625$ for $\kappa=10^{-8}\left[\mathrm{~m}^{2}\right]$. The overlap (of fixed thickness) is located in three different positions around the nominal interface $\Gamma$ of the sharp interface approach.

\begin{tabular}{ccccccc}
\hline overlap & \multicolumn{3}{c}{ Stokes domain } & \multicolumn{3}{c}{ Darcy domain } \\
& $e_{u}(\bar{x})$ & $e_{v}(\bar{x})$ & $e_{p}(\bar{x})$ & $e_{u}(\bar{x})$ & $e_{v}(\bar{x})$ & $e_{p}(\bar{x})$ \\
\hline lower & $2.628 \mathrm{e}-05$ & $7.469 \mathrm{e}-03$ & $1.154 \mathrm{e}-04$ & $4.884 \mathrm{e}-02$ & $4.314 \mathrm{e}-01$ & $1.111 \mathrm{e}-04$ \\
medium & $3.587 \mathrm{e}-03$ & $1.707 \mathrm{e}-02$ & $5.614 \mathrm{e}-03$ & $1.965 \mathrm{e}-02$ & $4.386 \mathrm{e}-01$ & $5.647 \mathrm{e}-03$ \\
upper & $7.220 \mathrm{e}-03$ & $3.173 \mathrm{e}-02$ & $1.133 \mathrm{e}-02$ & $3.050 \mathrm{e}-02$ & $4.362 \mathrm{e}-01$ & $1.137 \mathrm{e}-02$ \\
\hline
\end{tabular}

subdomains are $\Omega_{1}=(0,0.015) \times\left(z_{\Gamma}-\delta, 0\right)$ and $\Omega_{2}=(0,0.015) \times\left(-0.0075, z_{\Gamma}\right)$. The domain $\Omega_{1}$ represents a part of channel closed on the top side where the fluid can flow through the vertical sides, while $\Omega_{2}$ represents a vertical filter. The fluid is water, the porous medium will be characterized by its isotropic intrinsic permeability $\boldsymbol{\kappa}=\kappa \mathbf{I}$. We suppose that the fluid is subject to the gravitational force, thus $\mathbf{f}_{1}=\mathbf{f}_{2}=(0,-\rho g)^{t}\left[\mathrm{~kg} /\left(\mathrm{m}^{2} \mathrm{~s}^{2}\right)\right]$, where $\rho$ is the density of the water and $g$ the gravity acceleration. The fluid enters into the domain $\Omega_{1}$ through the vertical left-hand boundary, where we impose a parabolic inflow, on the top side of the domain $\Omega_{1}$ we set the no-slip boundary conditions $\mathbf{u}_{1}=\mathbf{0}$, while the fluid may leave the domain through the vertical right-hand boundary (denoted by $\partial \Omega_{1}^{N}$ ). Following the notations given in (2.1), the boundary data on $\partial \Omega_{1}^{N} \cup \partial \Omega_{1}^{D}$ can be summarized as follows: $\mathbf{u}_{1}=\mathbf{0}$ on $(0,0.015) \times\{0\}$ and on $\{0\} \times(-0.0055-\delta / 2,-0.005), \mathbf{u}_{1}=\left(-16 \cdot 10^{3} z(z+0.005), 0\right)^{t}$ on $\{0\} \times(-0.005,0)$, and $\mathbf{T}_{1}\left(\mathbf{u}_{1}, p_{1}\right) \cdot \mathbf{n}_{1}=9.8 \rho z \mathbf{n}_{1}$ on $\{0.015\} \times(-0.0055-\delta / 2,0)$.

Concerning the boundary conditions for the porous domain, we impose $\mathbf{u}_{2} \cdot \mathbf{n}_{2}=0$ on the vertical sides $\partial \Omega_{2}^{N}$ to represent that an impervious material is present outside the domain, while we set $p_{2}=-\rho g z$ on the bottom horizontal side $\partial \Omega_{2}^{D}$ to account for the presence of a stationary fluid below the porous domain. The amount of flux filtering through the interface depends on the permeability of the porous media and on the boundary data imposed on the bottom horizontal side on the pressure. According to the classification proposed by Levy and Sanchez-Palencia [35], this test is numbered among "near normal flows", for which $\nabla p_{2}$ has small projection on the nominal interface and the order of magnitude of $\nabla p_{2}$ is greater than that of $\nabla p_{1}$. To our knowledge, it has been neither experienced nor proved that either the BeaversJoseph (5.2) or the Beavers-Joseph-Saffman (5.1) 3 conditions correctly describe this coupling. However, the latter condition is used in the literature (see, e.g. [34, 24]). We aim at comparing the ICDD and SI-BJS approaches, studying both the quality of the solution and the associated computational costs. We consider several types of isotropic and homogeneous porous media characterized by different permeabilities.

In Figures 5.3 and 5.4 we show the hydrodynamic pressure $\tilde{p}_{2}=p_{2}+\rho g\left(z-z_{0}\right)$ and the velocity field corresponding to $\kappa=10^{-7}\left[\mathrm{~m}^{2}\right]$ and $\kappa=10^{-13}\left[\mathrm{~m}^{2}\right]$, respectively.

In Fig. 5.5 we plot the profiles of $u, v$, and $\tilde{p}$ at $\bar{x} \in\{3.75 e-3,7.5 e-3,1.125 e-2\}$ when $\kappa=10^{-7}$. Also in this test case we observe the close correspondence between the ICDD and SI-BJS solutions. A quantitative comparison of the two solutions provides differences that behave like in Test 1 (see Table 5.5) and shows that the tangential component of the Darcy velocity behaves like $\mathcal{O}(\kappa)$, while that of the Stokes velocity like $\mathcal{O}(\sqrt{\kappa})$, as in the case of "near parallel flows".

In Table 5.8 we report the number of ICDD iterations required to satisfy the stopping test on the residual up to a tolerance $\epsilon=10^{-9}$, the infimum of the cost 


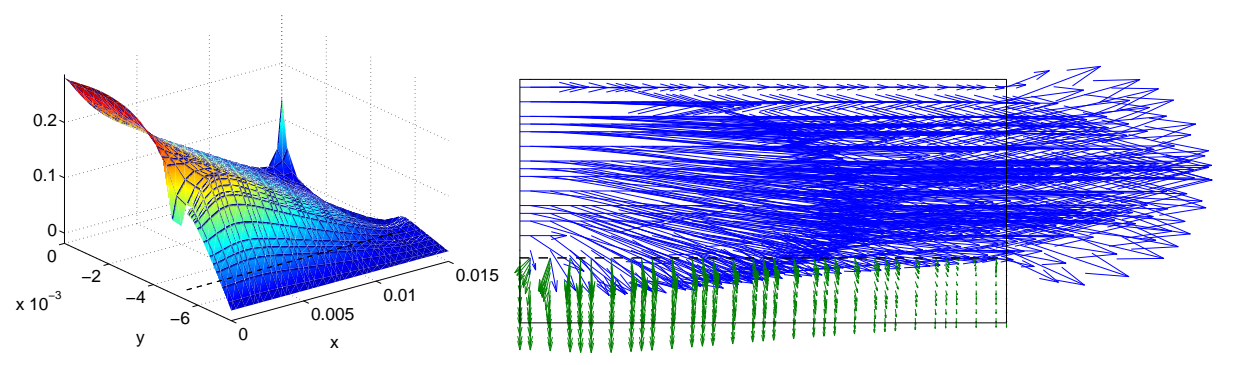

FIG. 5.3. Test 3. Hydrodynamic pressure and velocity field computed with $\kappa=10^{-7}\left[\mathrm{~m}^{2}\right]$ and $\delta=3 \cdot 10^{-4}$. For a better insight, the arrows in the right picture have been scaled by 10 .

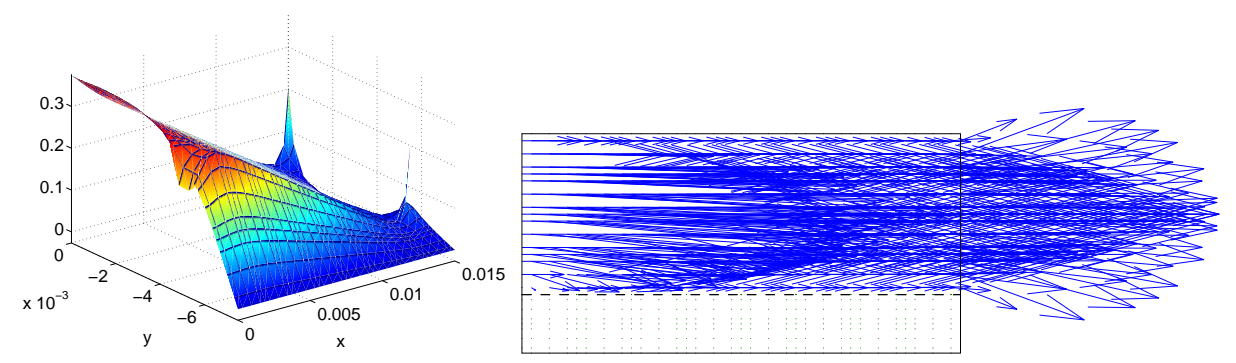

FIG. 5.4. Test 3. Hydrodynamic pressure and velocity field computed with $\kappa=10^{-13}\left[\mathrm{~m}^{2}\right]$ and $\delta=3 \cdot 10^{-7}$. For a better insight, the arrows in the right picture have been scaled by 10 .

functional $J_{h}$ attained at convergence, and the norms

$$
e_{\mathbf{u}, \Omega_{12}}=\left\|\mathbf{u}_{1}-\mathbf{u}_{2}\right\|_{L^{2}\left(\Omega_{12}\right)} /\left|\Omega_{12}\right|, \quad e_{p, \Omega_{12}}=\left\|p_{1}-p_{2}\right\|_{L^{2}\left(\Omega_{12}\right)} /\left|\Omega_{12}\right|,
$$

that measure the gap between Stokes and Darcy solutions on the overlap, normalized with respect to the size of the overlap (which is proportional to $\delta$ ). We see that the gap on the velocity decays as $\kappa$, while that on the pressure is independent of $\kappa$. $\delta$ is set according to (5.7), by choosing $\alpha_{B J}=1$. The discretization is almost fixed: stabilized $\mathbb{Q}_{4}-\mathbb{Q}_{4} h p-$ FEM for both Stokes and Darcy equations. Outside the overlap, the mesh is almost uniform, with grid size $h=2 \cdot 10^{-3}$ and $p=4$. The overlap is discretized by one strip of flattened quad elements of size $h \times \delta$. In Table 5.8, we also report the number of iterations required by Bi-CGstab to solve the Steklov-Poincaré equation associated with the Sharp Interface formulation of the Stokes-Darcy coupling with interface conditions (5.1), preconditioned by either the local Stokes SteklovPoincaré operator $\left(S_{s}\right)$ or the local Darcy Steklov-Poincaré operator $\left(S_{d}\right)$ (see [17]). The convergence rate of ICDD is clearly less sensitive to the permeability than that of the SI-BJS approach and it does not require designing a suitable preconditioner.

Computational cost, comparison with the sharp interface approach. As said above, one ICDD iteration corresponds to one Bi-CGStab iteration to solve the optimality system (4.15)-(4.19). Thus, it requires two matrix vector products (MVP), and each MVP requires solving two Stokes problems (like (4.15) and (4.17)) and two Darcy problems (like (4.16) and (4.18)).

Also the Steklov-Poincaré equation associated with the sharp interface approach can be solved by a Preconditioned Bi-CGstab method. In this case one MVP costs one Stokes plus one Darcy problem, while using the preconditioner costs one Stokes or one Darcy problem. By comparing the number of iterations of Table 5.8, and in 
TABLE 5.8

Test 3. ICDD iterations number, infimum of the cost functional, and errors on the overlap for different permeabilities. SI-BJS iterations number. Stabilized $\mathbb{Q}_{4}-\mathbb{Q}_{4}$ discretization in both subdomains.

\begin{tabular}{c|ccccc|cc}
\hline & \multicolumn{5}{|c|}{ ICDD } & \multicolumn{2}{c}{ SI-BJS \#it } \\
$\kappa\left[m^{2}\right]$ & $\delta$ & $\#$ it & $\inf J_{h}$ & $e_{\mathbf{u}, \Omega_{12}}$ & $e_{p, \Omega_{12}}$ & $P=S_{s}$ & $P=S_{d}$ \\
\hline $1 . e-7$ & $3 . e-4$ & 8 & $1.02 e-17$ & $1.49 e+0$ & $5.47 e-4$ & 13 & 17 \\
$1 . e-9$ & $3 . e-5$ & 5 & $6.49 e-20$ & $3.24 e-2$ & $6.07 e-4$ & 32 & 5 \\
$1 . e-11$ & $3 . e-6$ & 5 & $3.09 e-20$ & $3.22 e-4$ & $8.23 e-4$ & 33 & 4 \\
$1 . e-13$ & $3 . e-7$ & 5 & $4.29 e-20$ & $3.23 e-6$ & $3.94 e-4$ & 33 & 4 \\
\hline
\end{tabular}
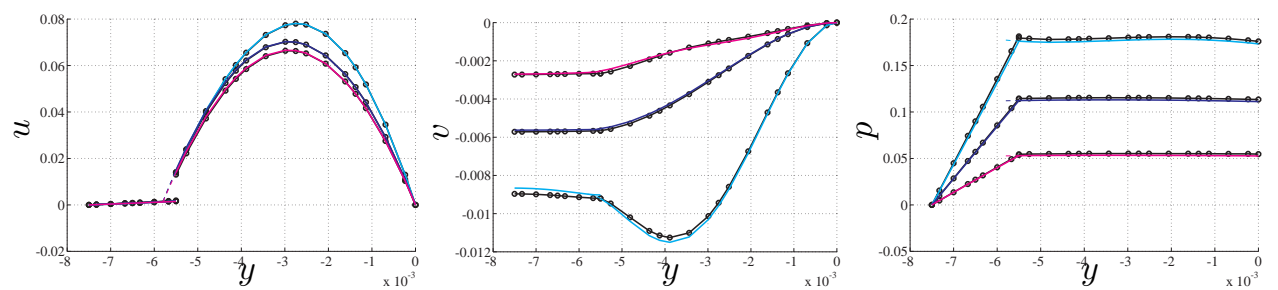

FIG. 5.5. Test 3. Profiles of u (left), $v$ (center), and hydrodynamic pressure $\tilde{p}$ (right), for the SI-BJS solution (black line) and the ICDD one (colored lines) at $\bar{x}=3.75 e-3$ (light blue), $\bar{x}=7.5 e-3$ (dark blue), $\bar{x}=1.125 e-2$ (magenta) when $\boldsymbol{\kappa}=10^{-7} \mathbf{I}$. The thickness overlap for $I C D D$ is $\delta=3 \cdot 10^{-4}$

view of the fact that one ICDD iteration costs a little more than one SI-BJS iteration, we conclude that the computational costs of ICDD and SI-BJS are comparable.

Nevertheless, the memory storage required by ICDD is less than the one required by SI-BJS. As a matter of fact, in ICDD, the Stokes matrices are the same for both primal and dual problems (and the same happens for Darcy), while in the case of SI-BJS, the preconditioner (either Stokes or Darcy) does not coincide with the matrix of the primal problem, in view of the different boundary conditions at the interface that characterize the direct local Steklov-Poincaré operator or its inverse (see [17] for a more detailed description of this approach).

Conclusions. In this paper the heterogeneous coupling between Stokes and Darcy equations by the ICDD method has been studied. The coupling has been formulated as an optimal control problem for which the well-posedness, the discretization and the convergence (of the discrete solution to the continuous one) have been analyzed. Several 2D test cases have shown both efficiency and robustness of this method, and that the ICDD solution is physically reasonable and comparable with the solution obtained by the classical Sharp Interface approach with Beavers--Joseph-Saffman condition (SI-BJS).

The ICDD method features several interesting properties that make it preferable to other existing coupling methods like the one based on the SI-BJS approach.

i) When the overlap thickness $\delta$ is chosen comparable to the characteristic length scale of the pores $\varepsilon \sim \sqrt{\kappa}$, the ICDD solutions are very close to the SI-BJS ones, for both "near parallel flows" (classical test case of BJ) and "near normal flows".

ii) The ICDD computational cost is comparable with that of SI-BJS, provided that the latter is preconditioned in an optimal way.

iii) Simplicity in handling Dirichlet interface conditions is another strong feature of ICDD, whereas interface conditions of SI-BJS involve both normal and tangential 
derivatives and they must accurately set up to take into consideration possible corners as well as non-straight interfaces.

iv) The convergence rate of ICDD iterations is independent of the discretization parameters. When the permeability is large, the number of iterations of ICDD is $\# i t \simeq \mathcal{O}\left(\delta^{q}\right)$ with $-1 \leq q \leq-1 / 2$. However, since $\delta \sim \sqrt{\kappa}$, the previous estimate is not penalizing, because when $\kappa$ is large, also $\delta$ is large.

Acknowledgments. The first author acknowledges funding from the European Union Seventh Framework Programme (FP7/2007-2013) under grand agreement no. 294229 .

\section{REFERENCES}

[1] G.S. Beavers and D.D. Joseph. Boundary conditions at a naturally permeable wall. J. Fluid Mech., 30:197-207, 1967.

[2] D. Boffi, F. Brezzi, and M. Fortin. Mixed Finite Element Methods and Applications. SpringerVerlag, 2013.

[3] H.C. Brinkman. A calculation of the viscous force exerted by a flowing fluid on a dense swarm of particles. Appl. Sci. Res. A, 1:27-34, 1947.

[4] C. Canuto, P. Gervasio, and A. Quarteroni. Finite-Element Preconditioning of G-NI Spectral Methods. SIAM J. Sci. Comput., 31(6):4422-4451, 2009/10.

[5] C. Canuto, M. Y. Hussaini, A. Quarteroni, and T. A. Zang. Spectral Methods. Fundamentals in Single Domains. Springer, Heidelberg, 2006.

[6] C. Canuto, M. Y. Hussaini, A. Quarteroni, and T. A. Zang. Spectral Methods. Evolution to Complex Geometries and Applications to Fluid Dynamics. Springer, Heidelberg, 2007.

[7] T. Carraro, C. Goll, A. Marciniak-Czochra, and A. Mikelić. Pressure jump interface law for the Stokes-Darcy coupling: confirmation by direct numerical simulations. J. Fluid Mech., 732:510-536, 2013.

[8] M. Chandesris and D. Jamet. Jump condition and surface-excess quantities at a fluid/porous interface: a multi-scale approach. Transp. Porous Med., 78:419-438, 2009.

[9] F. Cimolin and M. Discacciati. Navier-Stokes/Forchheimer models for filtration through porous media. Appl. Numer. Math., 72:205-224, 2013.

[10] P. Constantin and C. Foias. Navier-Stokes equations. The university of Chicago press, Chicago, London, 1988.

[11] M.R. Correa and A.F.D. Loula. A unified mixed formulation naturally coupling stokes and darcy flows. Comput. Methods Appl. Mech. Engrg., 198:2710-2722, 2009.

[12] H. Darcy. Les Fontaines Publiques de la Ville de Dijon. Dalmont, Paris, 1856.

[13] M. Discacciati. Domain Decomposition Methods for the Coupling of Surface and Groundwater Flows. PhD thesis, Ecole Polytechnique Fédérale de Lausanne, Lausanne (CH), 2004.

[14] M. Discacciati, P. Gervasio, and A. Quarteroni. Heterogeneous mathematical models in fluid dynamics and associated solution algorithms, volume 2040 of Lecture Notes in Mathematics, chapter 2, pages 57-123. Springer, 2011.

[15] M. Discacciati, P. Gervasio, and A. Quarteroni. The interface control domain decomposition (ICDD) method for elliptic problems. SIAM J. Control Optim., 51(5):3434-3458, 2013.

[16] M. Discacciati, P. Gervasio, and A. Quarteroni. The interface control domain decomposition (ICDD) method for the Stokes problem. J. Coupled Syst. Multiscale Dyn., 1(5):372-392, 2013.

[17] M. Discacciati and A. Quarteroni. Navier-Stokes/Darcy coupling: modeling, analysis, and numerical approximation. Rev. Mat. Complut., 22(2):315-426, 2009.

[18] H.I. Ene and E. Sánchez-Palencia. Équations et phénomènes de surface pour l'écoulement dans un modèle de milieu poreux. J. Mécanique, 14:73-108, 1975.

[19] L.P. Franca and S.L. Frey. Stabilized finite element methods: II. The Incompressible NavierStokes Equations. Comput. Meth. Appl. Mech. Engrg., 99:209-233, 1992.

[20] P. Gervasio, J.-L. Lions, and A. Quarteroni. Heterogeneous coupling by virtual control methods. Numerische Mathematik, 90(2):241-264, 2001.

[21] P. Gervasio and F. Saleri. Stabilized spectral element approximation for the Navier-Stokes equations. Numerical Methods for Partial Differential Equations, 14:115-141, 1998.

[22] D. Gilbarg and N.S. Trudinger. Elliptic partial differential equations of second order. Classics in Mathematics. Springer-Verlag, Berlin, 2001. Reprint of the 1998 edition. 
[23] R. Glowinski, Q.V. Dinh, and J. Periaux. Domain decomposition methods for nonlinear problems in fluid dynamics. Comput. Methods Appl. Mech. Engrg., 40(1):27-109, 1983.

[24] N.S. Hanspal, A.N. Waghode, V. Nassehi, and R.J. Wakeman. Development of a predictive mathematical model for coupled Stokes/Darcy flows in cross-flow membrane filtration. Chem. Eng. J., 149:132-142, 2009.

[25] T.J.R. Hughes, L.P. Franca, and M. Balestra. A new finite element formulation for computational fluid dynamics: V. Circumventing the Babuška-Brezzi condition: a stable PetrovGalerkin formulation of the Stokes problem accommodating equal-order interpolations. Comput. Meth. Appl. Mech. Engrg., 59:85-99, 1986.

[26] O. Iliev and V. Laptev. On numerical simulation of flow through oil filters. Computing and Visualization in Science, 6(2-3):139-146, 2004.

[27] O. Iliev and D. Vasileva. On a local refinement solver for coupled flow in plain and porous media. In Numerical Methods and Applications, volume 4310 of Lecture Notes in Computer Science, pages 590-598. Springer Berlin Heidelberg, 2007.

[28] A.S. Jackson, I. Rybak, R. Helmig, W.G. Gray, and C.T. Miller. Thermodynamically constrained averaging theory approach for modeling flow and transport phenomena in porous medium systems: 9. Transition region models. Advanced in Water Resources, 42:71-90, 2012.

[29] W. Jäger and A. Mikelić. On the boundary conditions at the contact interface between a porous medium and a free fluid. Ann. Scuola Norm. Sup. Pisa Cl. Sci., 23:403-465, 1996.

[30] W. Jäger and A. Mikelić. On the interface boundary condition of Beavers, Joseph and Saffman. SIAM J. Appl. Math., 60(4):1111-1127, 2000.

[31] W. Jäger, A. Mikelić, and N. Neuss. Asymptotic analysis of the laminar viscous flow over a porous bed. SIAM J. Sci. Comput., 22(6):2006-2028, 2001.

[32] I.P. Jones. Low Reynolds number flow past a porous spherical shell. Proc. Camb. Phil. Soc., 73:231-238, 1973.

[33] K. Khadra, P. Angot, S. Parneix, and J.-P. Caltagirone. Fictitious domain approach for numerical modelling of navier-stokes equations. International Journal for Numerical Methods in Fluids, 34(8):651-684, 2000.

[34] W.J. Layton, F. Schieweck, and I. Yotov. Coupling fluid flow with porous media flow. SIAM J. Numer. Anal., 40(6):2195-2218 (2003), 2002.

[35] T. Levy and E. Sánchez-Palencia. On boundary conditions for fluid flow in porous media. Internat. J. Engrg. Sci., 13(11):923-940, 1975.

[36] J.-L. Lions. Optimal Control of Systems Governed by Partial Differential Equations. SpringerVerlag, New York, 1971.

[37] J.-L. Lions and O. Pironneau. Algorithmes parallèles pour la solution de problèmes aux limites. C. R. Acad. Sci. Paris Sér. I Math., t. 327:947-952, 1998.

[38] A. Masud and T.J.R. Hughes. A stabilized mixed finite element method for Darcy flow. Comput. Methods Appl. Mech. Engrg., 191(39-40):4341-4370, 2002.

[39] E. Miglio, A. Quarteroni, and F. Saleri. Coupling of free surface and groundwater flows. Computers \& fluids, 32:73-83, 2003.

[40] A. Ochoa-Tapia and S. Whitaker. Momentum transfer at the boundary between a porous medium and a homogeneous fluid I. Theoretical development. Int. J. Heat Mass Transfer, 38:2635-2646, 1995.

[41] A. Ochoa-Tapia and S. Whitaker. Momentum transfer at the boundary between a porous medium and a homogeneous fluid II. Comparison with experiment. Int. J. Heat Mass Transfer, 38:2647-2655, 1995.

[42] A. Quarteroni. Numerical Models for Differential Problems. 2nd edition. Series MS\&A, Vol. 2. Springer, Milano, 2013.

[43] A. Quarteroni and A. Valli. Numerical Approximation of Partial Differential Equations. Springer Verlag, Heidelberg, 1994.

[44] A. Quarteroni and A. Valli. Domain Decomposition Methods for Partial Differential Equations. The Clarendon Press, Oxford University Press, New York, 1999.

[45] B. Rivière and I. Yotov. Locally conservative coupling of Stokes and Darcy flows. SIAM J. Numer. Anal., 42(5):1959-1977, 2005.

[46] P.G. Saffman. On the boundary condition at the interface of a porous medium. Stud. Appl. Math., 1:93-101, 1971.

[47] M. Sahraoui and M. Kaviany. Slip and no-slip velocity boundary conditions at interface of porous, plain media. International Journal of Heat and Mass Transfer, 35:927-943, 1992.

[48] H.A. van der Vorst. Iterative Krylov methods for large linear systems. Cambridge University Press, Cambridge, 2003. 